\title{
CHOOSING THE RIGHT BUSINESS PROCESS MATURITY MODEL.
}

\author{
Amy Van Looy ${ }^{a, b}$, Manu De Backer ${ }^{\mathrm{a}, \mathrm{b}, \mathrm{c}, \mathrm{d}}$, Geert Poels ${ }^{\mathrm{b}}$, Monique Snoeck ${ }^{\mathrm{d}}$ \\ a Faculty of Business Administration and Public Administration, Department of Management and Informatics, University College Ghent, Henleykaai 84, \\ B-9000 Ghent, Belgium \\ b Faculty of Economics and Business Administration, Department of Management Information Science and Operations Management, Ghent University, \\ Ghent, \\ Belgium \\ C Faculty of Applied Economics, Department of Management Informatics, University of Antwerp, Antwerp, Belgium \\ d Faculty of Business and Economics, Department of Management Informatics, K.U. Leuven, Leuven, Belgium
}

(This paper has been published in Information \& Management, 2013, 50 (7)).

\begin{abstract}
We built and tested a decision tool that organisations can use to properly select one business process maturity model (BPMM) out of many maturity models. This prototype consists of a novel questionnaire with decision criteria for BPMM selection, linked to a unique data set of 69 BPMMs. 14 criteria were defined by an international Delphi study, and weighed by the Analytical Hierarchy Process. Case studies showed (non-)profit and academic applications. Our purpose was to describe criteria enabling an informed BPMM choice (conform to decision-making theories, instead of ad hoc). We also propose a design process for building BPMM decision tools.
\end{abstract}

\section{Keywords}

Maturity model; business process; process improvement; decision support system; Delphi technique; Analytical Hierarchy Process

\section{Introduction}

Business processes describe how organisations operate, and therefore impact how organisations perform. Due to higher performance challenges and IT opportunities [32], business process maturity models (BPMMs) are increasingly important to help organisations obtain mature (or excellent) business processes [9]. As from the 1970s, maturity models are recognised as important improvement tools for organisations. Meanwhile, dozens of BPMMs have been designed [25], like CMMI [103] or OMG-BPMM [86]. They are evolutionary tools to systematically assess and improve capabilities (i.e. skills or competences) to reach business (process) excellence [29]. For instance, a BPMM may assess how capable an organisation is in modelling its processes or in running its processes without errors.

The huge number of BPMMs raises questions about their substantial differences. Some comparative studies have been made, albeit with a small number of BPMMs [14]. To our knowledge, the BPMM literature is mainly restricted to a design perspective by creating a theory to design BPMMs or by designing particular BPMMs, as in de Bruin and Rosemann [55]. Mettler [16] presents design criteria for maturity models from both a developer's and user's perspective, although not specific to the BPMM context and without an overview of existing models. Röglinger, Pöppelbuss and Becker [22] propose design criteria for BPMMs in particular. They present a limited BPMM overview to illustrate their criteria, but without practical advice on BPMM selection. Consequently, organisations and academics have no comprehensive overview of academic and industry-owned BPMMs and have an incomplete state of knowledge on how to select a BPMM that best fits their (organisational or research) needs. Therefore, this article helps users see the wood for the trees.

\section{RQ. Which criteria help users (i.e. organisations or academics) choose a BPMM?}

Our objective is to advance knowledge on criteria enabling a well-advised BPMM choice (conform to decision-making theories, instead of ad hoc). The identification of the most relevant criteria should result in a practical decision tool to make an informed BPMM choice out of a large BPMM sample. The criteria are identified by addressing key questions and trade-offs faced by many organisations, consultants, and scholars, and are then used to design a decision tool to recommend the most appropriate BPMM (out of the numerous available models) depending on individual needs. Our 
research distinguishes from existing studies by: (i) identifying a diverse set of BPMMs (in response to the lacking BPMM overview), (ii) identifying the most decisive selection criteria (in response to the lacking knowledge on BPMM selection), and (iii) designing a decision tool based on these criteria. We provide theoretical contributions by filling these important gaps, and by extending the literature with a thorough design process resulting in more informed decision-making for users of the BPMM decision tool. The major managerial implication is that the tool helps each organisation choose a BPMM that will best suit its particular needs.

The theoretical background of this problem statement is explained in section 2. Next, section 3 proposes our solution on which we elaborate in the methodology section (section 4). Afterwards, we explain how the decision tool was built (section 5) and tested (section 6). Section 7 discusses the tool as a solution to our research problem. We conclude by summarising the contributions and limitations (section 8), and main findings (section 9).

\section{Theoretical background}

Despite the many maturity models, a common conceptualisation is still lacking [17]. Hence, Figure 1 introduces the conceptual model of a BPMM for this study.

\section{(Take in Figure 1)}

As its name implies, a BPMM assesses and improves business process maturity. It does so by defining a number of maturity levels, each representing a higher or lower degree of maturity. The highest maturity level represents business process excellence. As shown in Figure 1, each maturity level covers a number of concrete capability areas (or areas of related capabilities) in which a business process can perform at a particular capability level. Van Looy, De Backer and Poels [30] present a framework of process capability areas with six main areas decomposed in 17 sub areas. The main capability areas are business process (i) modelling, (ii) deployment, (iii) optimisation, (iv) management, and a process-oriented (v) culture, and (vi) structure. The authors argue that BPMMs do not always address all capability areas, but that three clusters exist: (i) BPMMs limited to the first four basic areas in the traditional business process lifecycle [35], (ii) BPMMs combining the basic areas with a process-oriented culture, and (iii) BPMMs addressing the basic areas plus a process-oriented culture and structure. This classification is adopted in section 5.1.2 and is referred to as the 'modelType' of a BPMM in Figure 1.

Furthermore, Figure 1 explains that a BPMM can assess the current maturity level of a particular business process or a set of business processes, and suggest improvements to reach the next, desired maturity level. Similarly, a BPMM can assess and improve each capability area separately by capability levels and will sometimes also associate certain capability levels with a particular maturity level. Hence, maturity levels indicate the overall growth through all capability areas, whereas capability levels indicate the growth per capability area. The labels of maturity levels and capability levels may refer to (i) business process optimisation (e.g. initial, managed, standardised, predictable, innovating) [86], (ii) business process management (BPM) (e.g. BPM initiation, BPM evolution, BPM mastery) [97], or (iii) business process integration (e.g. ad hoc, defined, linked, integrated) [83]. Also their number of levels varies (e.g. five, three and four).

Further on, de Bruin and Rosemann [55] distinguish two types of BPMMs, also included as 'modelType' in Figure 1: models that assess (i) maturity of (one or more) specific business process(es), and (ii) maturity of BPM in general (i.e. of all business processes in the organisation). For instance, an organisation with ten business processes can choose between: (i) a BPMM that assesses and improves each process separately, (ii) a BPMM that assesses and improves the organisation's mastery in BPM, or (iii) a BPMM that combines both alternatives. This refinement may, for instance, 
indicate that the organisation is generally capable of modelling its processes, but that only some processes already have a graphical design, or that support processes do not need the same maturity level as core processes.

Examples of potential decision criteria, derived from the above, are the capability coverage of BPMMs, the labelling and number of maturity levels and capability levels, or the number of business processes addressed. This study will investigate which other criteria might guide BPMM selection, and which set of decision criteria is most relevant. Besides relevant decision criteria, a sound BPMM decision tool copes with requirements of decision-making theories, which explain how a motivated decision must be taken. We consider the following theories which underlie our solution (Table 1).

- Theory of bounded rationality [26]: as rational as possible given the limited human capabilities to capture data, resulting in optimal and satisfactory decisions.

- Theory of information symmetry [1]: with users having the same information as designers, resulting in better decisions.

- Theory of managerial work [18]: within the work rhythm of managers.

The multi-attribute utility theory [10] is an illustration of bounded rationality by decomposing a problem into smaller parts to avoid cognitive overload. In the context of our problem, it assumes that decision-makers use two or more criteria (or attributes) to choose a BPMM out of a set of alternatives to maximise utility. Therefore, the relative importance of criteria must be judged, which is subject to behavioural biases of individuals [4] or group collaboration [5]. Based on these theoretical backgrounds, a solution is proposed.

\section{Proposed solution}

Various authors assert that two research paradigms dominate the domains of applied sciences, such as BPM, operations management, and information systems (IS) [7]:

- the behavioural-science paradigm for building/testing theories to explain/predict situations;

- the design-science paradigm for building/testing artefacts to solve problem situations.

Our research is situated in the second paradigm, as other research on developing novel decision support systems [34]. We will rely on: (i) the IS design guidelines of Hevner et al. [7], (ii) the IS artefact types of March and Smith [15], and (iii) the IS design theory components of Walls et al. [33].

Table 1. Applying the design theory components of Walls et al. [33] (i.e. left column) to our study (i.e. right column).

\begin{tabular}{|c|c|}
\hline \multicolumn{2}{|l|}{ 1. Design product } \\
\hline $\begin{array}{l}\text { 1.1 Meta-requirements } \\
\text { (= goals to which the theory } \\
\text { applies) }\end{array}$ & $\begin{array}{l}\text { - Utility. The decision tool should enable an informed BPMM choice, which fits the needs. } \\
\text { - Effectiveness. The decision tool should be based on a limited set of the most relevant decision criteria. } \\
\text { - Efficiency. The decision tool should be quick to use (i.e. less than one hour). } \\
\text { - Quality. The output must be a clear and brief advice regarding which BPMM fits best. }\end{array}$ \\
\hline $\begin{array}{l}\text { 1.2 Meta-design } \\
\text { (= artefacts to meet the } \\
\text { meta-requirements) }\end{array}$ & $\begin{array}{l}\text { Artefact types conform to March and Smith [15]: } \\
\text { - conceptual model of the BPMM decision tool (constructs) } \\
\text { - overview of decision criteria and their weights (model) } \\
\text { - questionnaire that operationalises the decision criteria (model) } \\
\text { - decision table that applies the questionnaire and selects the best fit BPMM (method) } \\
\text { - decision tool that implements the decision table (resulting instantiation, to which all requirements apply) }\end{array}$ \\
\hline $\begin{array}{l}1.3 \text { Kernel theories } \\
\text { (= theories for the } \\
\text { requirements) }\end{array}$ & $\begin{array}{l}\text { - Theory of bounded rationality [26] => particularly utility and effectiveness required } \\
\text { - Theory of information symmetry }[1] \Rightarrow>\text { also quality required } \\
\text { - Theory of managerial work }[18] \Rightarrow>\text { also efficiency required } \\
\text { - Multi-attribute utility theory }[10] \Rightarrow>\text { particularly utility and effectiveness required }\end{array}$ \\
\hline $\begin{array}{l}\text { 1.4 Testable design product } \\
\text { hypotheses } \\
\text { (=meta-design satisfies the } \\
\text { meta-requirements?) }\end{array}$ & $\begin{array}{l}\text { - Utility. Organisations are more satisfied with the chosen BPMM if the decision tool is used, than if the } \\
\text { decision tool is not used (Req1). } \\
\text { - Effectiveness. The decision tool results in at least one BPMM. If more BPMMs are obtained, additional } \\
\text { information is given (Req2). } \\
\text { - Efficiency. Organisations are satisfied with the time and effort needed to use the decision tool (Req3). } \\
\text { - Quality. Organisations are satisfied with the clarity of the tool's output, i.e. the advised BPMM (Req4). }\end{array}$ \\
\hline \multicolumn{2}{|l|}{ 2. Design process } \\
\hline $\begin{array}{l}\text { 2.1 Design method } \\
\text { (= procedures for artefact } \\
\text { construction) }\end{array}$ & $\begin{array}{l}\text { - Content analysis of existing BPMM design documents }=>\text { conceptual model of BPMM decision tool and } \\
\text { how this can be instantiated for different BPMMs by a decision table }\end{array}$ \\
\hline
\end{tabular}




\begin{tabular}{|c|c|}
\hline & $\begin{array}{l}\text { - Consensus-seeking decision-making (i.e. Delphi) }=>\text { overview of criteria }+ \text { formulation of questionnaire } \\
\text { - Multi-criteria decision-making (i.e. AHP) }=>\text { overview of weighed criteria }+ \text { sequence in questionnaire } \\
\text { - Decision table design }=>\text { decision table } \\
\text { - Programming }=>\text { decision tool }\end{array}$ \\
\hline $\begin{array}{l}2.2 \text { Kernel theories } \\
\text { (= theories for the design } \\
\text { process) }\end{array}$ & $\begin{array}{l}\text { - Multi-attribute utility theory }[10] \Rightarrow \text { Delphi } \\
\text { - Theory of collaboration }[5] \Rightarrow \text { Delphi } \\
\text { - Theory of judging relative importance }[4] \Rightarrow \text { AHP }\end{array}$ \\
\hline $\begin{array}{l}2.3 \text { Testable design process } \\
\text { hypotheses } \\
\text { (=method satisfies the meta- } \\
\text { design?) }\end{array}$ & $\begin{array}{l}\text { - Experts are satisfied with the criteria (Req5) and weights (Req6). => overview of criteria and weights } \\
\text { - Organisations are satisfied with the criteria (Req7), their question formulation (Req8), and their sequence } \\
\text { (Req9). } \Rightarrow>\text { overview of criteria and weights, questionnaire, decision table } \\
\text { - The design guidelines of Hevner et al. [7] are met (Req10). } \Rightarrow>\text { all artefact types }+ \text { viable decision tool }\end{array}$ \\
\hline
\end{tabular}

Design research also builds and tests theories of designing artefacts and is thus different from pure development work [7;33]. For instance, Table 1 illustrates how we build a design theory for a BPMM decision tool by formulating hypotheses regarding the design product (i.e. working tool) and the design process (i.e. methodology). As hypotheses in the design-science paradigm rather refer to design choices or solution requirements than explanations (for decision-making), the remainder of this article will refer to requirements. These requirements will be used to discuss our solution in section 7 .

\subsection{Proposed solution requirements}

Due to the lack of any selection support for maturity models, we cannot evaluate our tool with respect to similar tools. Instead, our solution requirements are motivated by decision-making theories.

The bounded rationality theory of Simon [26] criticises classical decision-making theories as people are not omniscient. Instead, decision-makers must act as rational as their limited capabilities permit them to do. The multi-attribute utility theory [10] specifies that such informed decisions are achieved by considering a set of the most decisive criteria, in order to find a balance between an information overload (i.e. too many criteria) and ad hoc decisions (i.e. too few criteria). By answering this set, the alternative solutions are screened to verify which one best meets particular needs. Thus, we propose that BPMM selection must examine existing BPMMs on a limited set of predominant criteria, enabling an optimal and satisfactory choice without information overload:

Req1. Organisations are more satisfied with the chosen BPMM if the decision tool is used, than if the decision tool is not used.

Req2. The decision tool results in at least one BPMM. If more BPMMs are obtained, additional information is given.

Furthermore, in the theory of managerial work, Mintzberg [18] asserts that managers perform a great quantity of varied activities in small time periods. As each activity usually takes less than nine minutes and only a few exceed one hour, overburdened managers seek meaningful help. Hence, we posit that BPMM selection must fit within the work rhythm of managers:

Req3. Organisations are satisfied with the time and effort needed to use the decision tool.

Further on, the theory of information symmetry [1] proclaims that if buyers and users have similar information on product attributes, buyers tend to value a product closer to reality. As more accurate product valuations result in better decisions, potential BPMM users must acquire clear and brief advice on which BPMM best fits their purpose. This leads to:

Req4. Organisations are satisfied with the clarity of the tool's output, i.e. the advised BPMM.

On the other hand, the design process relies on the multi-attribute utility theory [10] by deriving the most relevant criteria in a Delphi study, i.e. a longitudinal panel study aiming at consensus. This panel must be carefully selected, and remains anonymous during the study to avoid group pressures, as stipulated by the theory of collaboration [5]. The Delphi panel will also weigh the final criteria by using the Analytical Hierarchy Process. Specific attention is needed to aggregate individual weights, as the theory of judging relative importance warns for individual biases [4]. Thus, we propose:

Req5. Delphi experts are satisfied with the criteria.

Req6. Delphi experts are satisfied with the weights of criteria. 
To be of use by organisations, the final criteria will be translated into a practical questionnaire, with criteria of higher weights appearing first. Not only the Delphi experts (during tool building), but also the users (during tool testing) must be satisfied with the obtained criteria and weights. Therefore:

Req7. Organisations are satisfied with the criteria.

Req8. Organisations are satisfied with the question formulation of criteria.

Req9. Organisations are satisfied with the sequence of criteria.

Finally, the established guidelines of Hevner et al. [7] assist in understanding, conducting and assessing research on viable information systems, like decision support systems. Based on this:

Req10. The design guidelines of Hevner et al. [7] are met.

\subsection{Proposed artefacts}

March and Smith [15] distinguish four IS artefact types, or design research outputs:

- construct: a conceptualisation or vocabulary to describe problems and solutions;

- model: a description of problems and solutions, based on the conceptualisation;

- method: a set of steps (i.e. algorithms or guidelines) to perform a task;

- instantiation: the realisation of constructs, models and methods in a working tool.

Translated to our research, the constructs of the BPMM decision tool are shown in Figure 2.

\section{(Take in Figure 2)}

On the right, Figure 2 indicates that our decision tool will be based on a set of relevant decision criteria with corresponding options. They are a priori identified by subject-matter experts (through consensus-seeking decision-making). Figure 2 also shows that the experts will weigh the selected criteria and options (through multi-criteria decision-making). These relative weights will allow evaluating existing BPMMs with a final score (prior to tool development), and will determine the sequence in which criteria are presented in the tool. Regarding this presentation, the criteria and options will be translated into questions and answer options, as visualised on the left of Figure 2. By filling out the questionnaire (i.e. selecting for the organisation the appropriate answer options for the questions that operationalise the criteria), the tool will present the BPMMs that best match the responses.

Regarding the other artefact types of March and Smith [15], two models will be designed: (i) an overview of the final decision criteria and options, including their weights, and (ii) a questionnaire. A decision table will be designed as a method to process the questionnaire. It will explain how answers suggest a BPMM choice. The instantiation will be the physical BPMM decision tool that organisations can use to choose a BPMM that fits their needs.

Besides tool development, we intend to build a design process (or methodology) for building BPMM decision tools. This process should allow increasing the number of BPMMs to choose from without having to re-implement the decision tool. Similarly, the design process that we followed should be replicable, for example, when a content analysis of newly developed BPMMs reveals the possibility of new decision criteria to take into account.

\section{Methodology}

\subsection{Initial list of decision criteria}

In the second quarter of 2010, we sampled 69 BPMMs as candidate outputs for our BPMM decision tool. 32 BPMMs address specific process types (24 for supply chains and 8 for collaboration processes), whereas 37 BPMMs are generic and can be applied to any process type (Appendix A). We initially searched for articles in academic databases (i.e. SCI-Expanded, SSCI, A\&HCI, CPCI-S, 
CPCI-SSH, BPM Journal) and non-academic search engines (i.e. Google, Google Scholar) by using the combined keywords of 'process' and 'maturity'. Then, we traced the references in identified articles for other relevant sources. Models were included if they: (1) present maturity or capability levels, and (2) primarily focus on improving processes instead of other organisational assets.

The search for decision criteria started in the BPMM literature, limited to design criteria as BPMM characteristics. A qualitative content analysis [12] of the sampled BPMMs resulted in 20 potential decision criteria. However, not all design criteria might be relevant when choosing a BPMM and also other criteria may become prominent (e.g. financial or practical considerations). Hence, four additional criteria were obtained from peer feedback on a conference on information systems (Eis) and a pilot study with BPM scholars. Finally, the criteria were reviewed throughout a Delphi study, as explained in the next section.

Content analysis is to some extent subjective, but multiple aspects guarantee objectivity or intersubjectivity. First, the official BPMM design documents were analysed instead of subjective conversations, feelings or opinions. Secondly, we collected multiple documents for the same BPMM, if possible. Thirdly, the first author was the main coder, and other researchers were consulted to obtain a reliable coding. The BPMM design documents were also repeatedly analysed over time from the third quarter of 2010 until the second quarter of 2012. For reasons of conciseness, we refer to particular BPMMs by IDs (Appendix A).

\subsection{Identifying decision criteria by consensus-seeking decision-making (i.e. Delphi)}

To acquire a more objective (i.e. inter-subjective) set of decision criteria, we consulted independent subject-matter experts in an international Delphi study. A Delphi study is a consensus-seeking decision-making method using 'a series of sequential questionnaires or rounds, interspersed by controlled feedback, that seek to gain the most reliable consensus of opinion of an expert panel' [3,p.458]. We have chosen a Delphi study as its iterative approach enhances validity, compared to a single round. According to Van De Ven and Delbecq [28], it generally results in a higher quantity and quality of ideas than other group decision-making methods. The experts remained anonymous during the study, which minimises group pressures [5]. Moreover, a Delphi study is widely used for exploring ideas and structuring group communication on framework development and rating [55;20].

Table 2. An overview of the Delphi method.

\begin{tabular}{|c|c|c|}
\hline Round & Input of codification panel & Output of expert panel \\
\hline $\begin{array}{l}1 \\
\text { Nov } \\
\text { Dec 2011) }\end{array}$ & $\begin{array}{l}\text { Phase 1: brainstorming } \\
\text { - Propose initial criteria } \\
\text { - Request missing criteria }\end{array}$ & $\begin{array}{l}\text { - Warm-up: capabilities } \\
\text { ○ Rate the importance per capability sub area [30] } \\
\text { - Per initial criterion } \\
\text { ○ Rate its importance for BPMM selection } \\
\text { - For all criteria together } \\
\text { O Rate their overall importance } \\
\text { - Missing criteria } \\
\circ \quad \text { Propose criteria that are not in the initial list }\end{array}$ \\
\hline $\begin{array}{lr}2 & \\
(\text { Dec } & 2011 \\
- & \text { Jan } \\
2012) & \end{array}$ & $\begin{array}{l}\text { Phase 2: narrowing down } \\
\text { Consolidate the criteria } \\
\text { considered important for } \\
\text { BPMM selection }\end{array}$ & $\begin{array}{l}\text { - Warm-up 1: capabilities } \\
\circ \quad \text { How must the criterion 'presence of capabilities' be questioned in the decision tool? } \\
\text { - Warm-up 2: relevance of BPMM selection } \\
\text { ○ Rate the importance of BPMM selection } \\
\text { - Per criterion } \\
\text { o Rate its importance for BPMM selection } \\
\text { - For all criteria together } \\
\circ \quad \text { Rate their overall importance }\end{array}$ \\
\hline $\begin{array}{l}3 \\
(\text { Jan }- \text { Feb } \\
2012)\end{array}$ & Phase 2: continued & $\begin{array}{l}\text { - Warm-up: capabilities } \\
\text { ○ Which question formulation regarding 'presence of capabilities' do you prefer? } \\
\text { - Per criterion } \\
\text { ○ Rate its importance for BPMM selection } \\
\text { - For all criteria together } \\
\text { ○ Rate their overall importance } \\
\text { - Wrap-up: further discussion? } \\
\text { o Do you prefer an additional round for discussing open criteria? }\end{array}$ \\
\hline $\begin{array}{l}4 \\
(\mathrm{Feb}\end{array}$ & $\begin{array}{l}\text { Phase 3: weighing } \\
\text { - Determine final criteria }\end{array}$ & $\begin{array}{l}\text { - Warm-up: capabilities } \\
\circ \quad \text { What would you use a BPMM for, and which capabilities correspond to such usage? }\end{array}$ \\
\hline
\end{tabular}




\begin{tabular}{|c|c|c|}
\hline $\begin{array}{l}\text { March } \\
\text { 2012) }\end{array}$ & $\begin{array}{l}\text { - Request weights for final } \\
\text { criteria }\end{array}$ & $\begin{array}{l}\text { - Weighing } \\
\circ \quad \text { Weigh criteria and options with consensus }\end{array}$ \\
\hline $\begin{array}{l}5 \\
\text { (April } \\
2012 \text { ) }\end{array}$ & $\begin{array}{l}\text { Wrap-up } \\
\text { - Summarise the study }\end{array}$ & $\begin{array}{l}\text { - For all criteria together } \\
\circ \quad \text { Rate your overall satisfaction with the weights }\end{array}$ \\
\hline
\end{tabular}

As shown in Table 2, we followed a 'modified Delphi' approach [19]. The initial list of 24 criteria was presented in the first round and missing criteria were requested in an open ended way. This contrasts with a 'classical Delphi' which only starts with open questions. However, our initial list ensured common ground among experts. Hsu and Sandford [8] asserts that this way of working is easier for experts and coders, and ensures recognising criteria from previous research that otherwise might have been omitted.

\subsubsection{Expert panel}

A balance was sought between BPM representatives in academia and industry to stimulate normative discussions. The academics had credible BPM(M) publications in academic journals, and the practitioners designed a BPMM, applied BPM(M), or were interested in BPMM selection. The practitioners were consultants and managers with decision power in large to medium-sized organisations, both profit and non-profit. Secondarily, we aimed to have at least one academic and one industry expert per continent to ensure global criteria for BPMM selection. Our intention is not to obtain a representation of the population or state of BPM practices within a given region. Instead, this procedure allows introducing different backgrounds to minimise bias [5].

Table 3. The expert panel by role and region.

\begin{tabular}{l|c|c|c}
\hline \multicolumn{1}{c|}{ Role / Region } & Academia & Industry & \\
\hline Africa & 1 & 1 & 2 \\
\hline Australasia & 1 & 4 & 3 \\
\hline Europe & 6 & 3 & 10 \\
\hline North America & 2 & 1 & 5 \\
\hline South America & 1 & 11 & 2 \\
\hline
\end{tabular}

The selection procedure conforms to [20]. 61 invitations were sent, of which 8 were referrals. 22 experts agreed to participate (Table 3). Mid-November 2011, the Delphi study started with 11 academics and 11 practitioners, each from five continents. This size corresponds to other Delphi studies, which often varies from 10 to 20 to facilitate group discussions with accuracy and low dropout rates $[20 ; 8]$.

\subsubsection{Codification panel}

The responses of the expert panel were anonymously analysed by a codification panel, consisting of the principal researcher as Delphi coordinator and three other academics (i.e. including one independent coder from another university to avoid a research bias). Therefore, the Delphi coordinator managed the random mapping of experts with unique identity codes (i.e. $\mathrm{R}+$ consecutive number). The questionnaires were analysed with SPSS in rounds 1 to 3, and MS Excel in round 4.

The coders stopped iterating a particular criterion when one of the three stopping conditions was reached [20;19]: (i) consensus was reached (defined by consensus conditions) to include a criterion in the tool or to exclude it, (ii) results for the criterion became repetitive (defined by stability conditions) before reaching consensus, to exclude a criterion, or (iii) experts were no longer willing to continue iterating (defined by fatigue conditions), in which case all remaining criteria without consensus were excluded.

\subsubsection{Consensus conditions}

For methodological soundness, our study combined four existing consensus conditions. When applied to ratings on a 7-point Likert scale (1=extremely unimportant; 7=extremely important), these complementary conditions are: 
- $50 \%$ of the experts must agree on the two most extreme scores (i.e. either 1-2 for consensus to exclude a criterion or 6-7 for consensus to include a criterion), and

- $75 \%$ must agree on the three most extreme scores (i.e. either 1-2-3 or 5-6-7), and

- the interquartile range (IQR, i.e. the distance between the $75^{\text {th }}$ and $25^{\text {th }}$ percentile, or the range of the middle $50 \%$ of responses for a criterion) must be 1.50 or less, and

- no opposite extreme score given by any expert (i.e. either no 7 or no 1 for a criterion), because 'if dissenters drop out, then there is artificial consensus' [19,p.43].

\subsubsection{Stability conditions}

Per criterion, responses were assumed repetitive between two Delphi rounds if:

- a significant positive association exists, measured by Spearman's rho and Kendall's tau-b $(0<$ rho and tau-b $=<1 ; \mathrm{p}<0.05$ ), and

- a significant level of agreement exists, measured by Cohen's Kappa ( $0.4=<\mathrm{kappa}=<1 ; \mathrm{p}<0.05)$.

To examine opinion changes (i.e. between unimportance 1-2-3, neutral 4 and importance 5-6-7), we recoded the 7-point scale into a 3-point scale before measuring stability.

\subsubsection{Fatigue conditions}

Besides consensus and stability, the study stopped if the majority of experts indicated they were no longer willing to continue iterating. Hasson et al. [6] and Mullen [19] propose a response rate of at least $70 \%$ compared to the initial panel size in order to maintain research rigour and validity.

\subsection{Weighing decision criteria by multi-criteria decision-making (i.e. AHP)}

The experts weighed the decision criteria that reached consensus, including their options. A possible criterion is the 'number of business processes'. The possible answer options to be weighed are 'one', 'more', 'all' business processes, or a 'combination'. We recall that weights will determine the sequence of criteria in the decision tool, but also allow a critical view on the many BPMMs by calculating selection scores before launching the final decision tool. BPMMs evaluated with the lowest scores will be omitted from the collected BPMM sample to guarantee the quality of candidate BPMMs. Given this purpose, weighing implies eliciting the relative importance of selected criteria and options.

Three common ranking methods in Delphi studies are: (i) simple rankings (e.g. item A > item B), (ii) ratings on a Likert scale, as used in the other Delphi rounds (e.g. item $A=6 / 7$ and item $B=3 / 7$ ), and (iii) pairwise comparisons (e.g. item A is three times more/less important than item B). Only the third method calculates relative importance. For making pairwise comparisons, we applied a multi-criteria decision-making method, as many decision support systems do [11]. The Analytical Hierarchy Process (AHP) [24] was chosen without requiring consensus, i.e weights result from geometrically averaging consistent responses of the experts.

Among multi-criteria decision-making methods, AHP is widely used for three decades. AHP has also been previously integrated in Delphi studies, either throughout the rounds [2] or limited to the final round [27]. Compared to other multi-criteria decision-making methods [2], AHP structures the criteria in a hierarchical model and analyses how consistent the judgements of relative importance are.

AHP calculates relative weights based on judgement matrices and normalised principal Eigen vectors (i.e. priority vectors). A judgement matrix uses the typical AHP 9-point scale (1/9=extremely less important; $1=$ =equally important; $9=$ extremely more important) to describe how much more or less important each row item is compared to each column item. For instance, a $4 \times 4$ matrix for the criterion 'number of business processes' shows all answer options in the row and column headings: 'one', 'more', 'all', and a 'combination' of business processes. The experts would make six comparisons: 'one' to 'more', 'one' to 'all', 'one' to 'combination', 'more' to 'all', 'more' to 'combination', and 
'all' to 'combination'. Other entries are derived by transposing the matrix. The responses were collected in MS Excel, and afterwards analysed by a dedicated AHP tool: Super Decisions [23]. Besides a thorough calculation, AHP measures a consistency ratio (CR) per matrix. Suppose an expert indicates that answer option 'one' is three times more important than option 'more', and 'more' is four times more important than 'all'. Non-random answers would indicate that 'one' is five through nine times more important than 'all'. Examples of random answers are: 'one' is twice as important than 'all' (i.e. inconsistent degree on the AHP scale), or 'one' is seven times less important than 'all' (i.e. inconsistent direction). Our study only geometrically averaged non-random judgements $(\mathrm{CR} \leq 0.1)$.

\subsection{Decision table and questionnaire}

To build the decision tool, the selected criteria were translated into a questionnaire. Their application to the sample of 69 identified BPMMs was summarised into a decision table, allowing systematically and visually guiding through the questions (i.e. criteria). We recall that a decision table design does not take into account the a priori selection scores, but that weights were used to propose a sequence of questions, i.e. criteria with higher weights appear first in the questionnaire. The decision table with the proposed sequence was constructed by using the PROLOGA software tool [31]. The manual questionnaire and decision table were direct inputs to create an online version of the BPMM decision tool, developed conform to Figure 2. The tool dynamically creates a decision table based on the questions that are actually answered (i.e. in the proposed sequence or a sequence determined the user's particular needs). It is programmed in JavaScript with jQuery user interface templates and in PHP, supported by a relational MySQL database.

\section{Building the BPMM decision tool}

Our Delphi study is characterised by high response rates in all five rounds: $100 \%, 77.27 \%, 90.91 \%$, $77.27 \%$, and $90.91 \%$ (initial $\mathrm{N}=22$ ). Only two experts permanently dropped-out after round 1 due to other commitments, whereas all remaining experts continued participating. Only three experts missed round 2 (i.e. during the Christmas holidays) and another three did not participate in round 4 (i.e. because pairwise comparisons are more time-consuming). Nevertheless, the response rates never reached the limit of 70\% [6;19]. This section shows the Delphi results and the building of the tool.

\subsection{Warm-up discussions}

Each round was preceded by warm-up questions regarding the relevance of BPMM selection and the interpretation of capabilities (i.e. which are core) to orient experts to the study.

\subsubsection{Relevance of BPMM selection}

Open comments in the first round gave rise to opposing statements about BPMM selection.

- 'The choice of BPMM is secondary to its application. You can work with pretty much any BPMM. The secret is getting the organisation to recognise deficiencies, create willingness to act and to follow-through on your findings'.

- 'My overall project investment is likely to be $10 \%$ on BPMM selection and $90 \%$ on the actual process maturity assessment, so I don't want to start using a model and discover it is flawed/unusable after I start using it'.

Hence, in the second round, the experts rated the importance of BPMM selection.

\section{(Take in Figure 3)}

Figure 3 shows that $76.48 \%$ recognised the importance of selecting an appropriate BPMM (score 5-67). No respondent rated BPMM selection as extremely unimportant (score 1). Respondents indicating 
some degree of unimportance commented that assessing and improving business processes is core, and therefore more important. Nevertheless, the large majority commented that investing in BPMM selection can save money and efforts later on, and that choosing a fit for purpose is critical. Otherwise, a BPMM could result in frustrations, a too high workload or an impractical way of working.

\subsubsection{Presence of capabilities}

The second type of warm-up questions coped with business process capabilities, as explained in section 2. In the first round, the experts were asked to indicate which of the 17 capability sub areas in the framework of Van Looy et al. [30] must be addressed by a BPMM.

Table 4. The percentage of experts agreeing on capability sub areas for BPMM.

\begin{tabular}{l|l|l|l}
\hline Yes $(\%)$ & 17 capability sub areas & 6 main capability areas & 3 clusters \\
\hline 90.91 & Design & Modelling & \multirow{2}{*}{ Basic cluster } \\
\hline 90.91 & Analysis & Deployment & \\
\hline 81.82 & Implementation and enactment & & \\
\hline 95.45 & Measurement and control & & \\
\hline 81.82 & Evaluation & Management & \\
\hline 90.91 & Improvement & & \\
\hline 86.36 & Strategy and key performance Indicators & & \multirow{2}{*}{ Basic + Culture } \\
\hline 72.73 & External relationships and service level agreements & & \\
\hline 90.91 & Roles and responsibilities & Culture & \\
\hline 77.27 & Skills and training & & \multirow{2}{*}{ Basic + Culture +} \\
\hline 54.55 & Daily management & & Structure \\
\hline 90.91 & Values & Structure & \\
\hline 81.82 & Attitudes and behaviours & & \\
\hline 68.18 & Appraisals and rewards & & \\
\hline 90.91 & Top management commitment & & \\
\hline 50.00 & Organisation chart & & \\
\hline 95.45 & Governance bodies & & \\
\hline
\end{tabular}

Table 4 shows that a simple majority agreed on the importance of all capability sub areas (i.e. $50 \%$ or more), with an absolute majority for 13 sub areas (i.e. $75 \%$ or more). Some experts asked for a further aggregation, as (i) the list is too complex for practitioners new to BPMMs, and (ii) different maturity types [55] may require different subsets. Therefore, round 2 also presented the main capability areas of Van Looy et al. [30]. 58.82\% of the respondents agreed that the decision tool must consider main capability areas, compared to $23.53 \%$ for sub areas. Consequently, round 3 presented two question formulations. The first alternative listed the main areas as separate options, whereas the second alternative aggregated them into three clusters. The answers were distributed fifty-fifty. To collect different contexts for BPMM use, round 4 asked the experts what they would use a BPMM for, and which main capability areas correspond to such usage. All but one expert combined capability areas similar to the clusters in Table 4. This supports a question formulation with three clusters, and corroborates the framework of Van Looy et al. [30].

\subsection{Brainstorming}

In the first Delphi round, after the warm-up, a non-exclusive list of 24 criteria was presented:

(1) number of assessed organisations

(3) certification

(5) number of assessors

(7) business versus IT respondents

(9) number of assessment items

(11) rating scale

(13) number of business processes

(15) architecture type (i.e. staged with maturity levels or continuous with capability levels)
(2) lead assessor

(4) benchmarking

(6) functional role of respondents

(8) data collection technique

(10) assessment duration

(12) presence of capabilities

(14) type of business processes

(16) number of lifecycle levels (i.e. maturity levels or capability levels) 
(17) level calculation

(19) labelling of levels

(21) architecture details (i.e. level of guidance)

(23) validation methodology
(18) level representation

(20) external view of levels

(22) creation methodology

(24) direct costs to access and use a BPMM

Criteria 4, 22, 23, and 24 were proposed by peer feedback (section 4.1), whereas other criteria were derived from a content analysis of BPMM design documents. Only two missing criteria were added after round 1: (i) the purpose for which a BPMM is intended to be used (i.e. it replaces criteria 3 and 4), and (ii) whether assessment items are publicly available. The second round started with 24 criteria.

\subsection{Narrowing-down by consensus-seeking decision-making}

The experts narrowed down the 24 criteria into a critical set for BPMM selection. After three rounds, we stopped iterating as $75 \%$ of the respondents indicated they were no longer willing to continue iterating. 14 of the 24 criteria reached consensus of being important, and are included in the decision tool (Table 5, i.e. four criteria in round 1 , six criteria in round 2 and four criteria in round 3 ).

\section{Table 5. The final criteria for BPMM selection.}

\begin{tabular}{|c|c|c|}
\hline Round & Criterion & Definition \\
\hline 1 & Presence of capabilities & The business process capability areas to be assessed and improved. \\
\hline 1 & Number of business processes & The number of business processes to be assessed and improved. \\
\hline 1 & Type of business processes & $\begin{array}{l}\text { Whether the maturity model addresses specific process types (e.g. supply chains or } \\
\text { collaboration processes) or can be applied to any process type. }\end{array}$ \\
\hline 1 & Functional role of respondents & $\begin{array}{l}\text { The explicit recognition to include people from outside the assessed organisation(s) as } \\
\text { respondents. }\end{array}$ \\
\hline 2 & Purpose & The purpose for which the maturity model is intended to be used. \\
\hline 2 & Validation methodology & $\begin{array}{l}\text { Evidence that the maturity model is able to assess maturity and helps to enhance the efficiency } \\
\text { and effectiveness of business processes. }\end{array}$ \\
\hline 2 & Architecture type & The possibility to define a road map per capability and/or a road map for overall maturity. \\
\hline 2 & Architecture details & The degree of guidance that a maturity model gives on your journey towards higher maturity. \\
\hline 2 & Data collection technique & The way information is collected during an assessment. \\
\hline 2 & Rating scale & The type of data that is collected during an assessment. \\
\hline 3 & Assessment availability & $\begin{array}{l}\text { Whether the assessment items and level calculation are publicly available (instead of only } \\
\text { known to the assessors). }\end{array}$ \\
\hline 3 & Direct costs & The direct costs to access and use a maturity model. \\
\hline 3 & Number of assessment items & The maximal number of questions to be answered during an assessment. \\
\hline 3 & Assessment duration & The maximal duration of a particular assessment. \\
\hline
\end{tabular}

The ten criteria not reaching consensus did not show a bimodal distribution with academics opposed to practitioners. Most criteria were blocked due to a few experts with opposite opinions, but with an agreeing majority to include them. However, seven of them already became stable $(\mathrm{p}<0.05)$, and two were almost stable $(0.05<\mathrm{p}<0.10)$. Particularly, after round 2 , the "level calculation" and "level representation" were already stable without tendency towards consensus. The "methodology" criterion also became stable after round 2, but was iterated again because some consensus conditions became satisfied after a shift from neutral to positive opinions. After round 3, the only remaining criterion without stability $(\mathrm{p}>0.10)$ was the "number of assessed organisations". Until now, crossorganisational processes are mostly studied from the perspective of one organisation. It is likely that this criterion will increase in importance, along with the emergence of cross-organisational processes and supply chains.

\section{(Take in Figure 4)}

The overall importance for all criteria reached consensus as from the first round. Figure 4 shows that $86.37 \%$ of the respondents in round 1 agreed that the initial criteria were somewhat to extremely important (score 5-6-7), and 63.64\% indicated very to extremely important (score 6-7). Finally, both values increased to $95 \%$ in round 3 (i.e. with only two respondents less than round 1). The overall importance thus increased, with a high and stable median of 6 , and a decreased, negligible IQR of 0 . 
A consensus to exclude criteria could not be reached, which means that all criteria were considered important to some degree. This can partly be explained by our 'modified Delphi' approach. Another explanation is given by a respondent as: 'transparency is key', or all criteria must be sufficiently explained in the design documents before a BPMM can be properly used. For instance, even if a BPMM explains all 14 selected criteria, it only becomes usable if it also explains the less decisive criteria (e.g. how many maturity levels it contains, how maturity levels are calculated, who the assessors might be, etc.). This finding adds an interesting dimension to the research. Besides the planned selection score per BPMM based on the final criteria (Table 2), an opportunity arises to calculate a transparency score per BPMM across all criteria (i.e. whether each criterion is present in the BPMM design documents). We recall from sections 4.3 and 4.4 that scoring aims at evaluating the quality of the BPMM sample, but without affecting the tool's decision table.

\subsection{Ranking and weighing by multi-criteria decision-making}

To facilitate ranking and weighing, we grouped the final 14 criteria: (i) assessment criteria (i.e. how maturity is measured and by whom), (ii) improvement criteria (i.e. what is measured as maturity, particularly the capabilities and their improvements to reach the successive levels), and (iii) nondesign criteria (i.e. not directly related to assessment and improvement).

The weights were gradually assigned, following the hierarchical AHP model of Table 6. For reasons of conciseness, the hierarchical model refers to criteria by IDs (e.g. G2 for the group of improvement criteria and I05 for the 'number of business processes').

Table 6. The hierarchical model of weighed decision criteria for BPMM selection.

\begin{tabular}{|c|c|c|c|c|c|}
\hline \multicolumn{6}{|c|}{ O1 RATING THE SELECTION OF BPMMs (min. score: 0; max. score: 100) } \\
\hline \multicolumn{6}{|l|}{ Groups: } \\
\hline $\begin{array}{l}\text { G1 ASSESSMENT } \\
\text { CRITERIA }\end{array}$ & 30.46 & $\begin{array}{c}\text { G2 IMPROVEMENT } \\
\text { CRITERIA }\end{array}$ & 52.14 & $\begin{array}{c}\text { G3 NON-DESIGN } \\
\text { CRITERIA }\end{array}$ & 17.40 \\
\hline \multicolumn{6}{|l|}{ Criteria: } \\
\hline A01 Rating scale & 7.78 & I01 Presence of capabilities & 17.03 & N01 Purpose & 7.41 \\
\hline Qualitative & 3.57 & Modelling & 69 & Awareness & 7.18 \\
\hline Quantitative & 2.98 & Deployment & .54 & Benchmarking & 7.41 \\
\hline Both & 7.78 & Optimisation & .58 & Certification & 2.33 \\
\hline & & Management & 4.47 & & \\
\hline & & Culture & 2.84 & & \\
\hline & & Structure & 1.91 & & \\
\hline A02 Data collection technique & 7.44 & 102 Architecture type & \multirow{2}{*}{$\frac{10.55}{.28}$} & N02 Validation & 6.57 \\
\hline Objective & 3.58 & Continuous & & Application & 2.04 \\
\hline Subjective & 2.28 & Staged & 7.50 & Outcomes & \multirow[t]{2}{*}{6.57} \\
\hline Both & 7.44 & Both & 10.55 & & \\
\hline A03 Assessment duration & 3.91 & 103 Architecture details & 10.28 & N03 Costs & 3.42 \\
\hline Day & 1.63 & Descriptive & 5.93 & Free & 3.42 \\
\hline Week & 3.91 & Implicit prescriptive & 10.28 & Charged & 1.47 \\
\hline Longer & 1.41 & Explicit prescriptive & 5.84 & & \\
\hline A04 Assessment availability & 3.82 & I04 Type of business processes & 8.31 & & \\
\hline Fully known & 3.82 & Generic & 8.31 & & \\
\hline Partially known & 3.31 & Domain-specific & 4.44 & & \\
\hline Fully unknown & 1.27 & & & & \\
\hline $\begin{array}{l}\text { A05 Functional role of } \\
\text { respondents }\end{array}$ & 3.82 & $105 \mathrm{Nr}$. of business processes & 5.97 & & \\
\hline Only internal & 2.10 & One & 1.83 & & \\
\hline Also external & 3.82 & More & 4.35 & & \\
\hline & & All & & & \\
\hline & & Combination & & & \\
\hline A06 Nr. of assessment items & 3.69 & & & & \\
\hline $0-19$ & 1.66 & & & & \\
\hline $20-49$ & 3.69 & & & & \\
\hline $50-99$ & 3.10 & & & & \\
\hline $100-299$ & 1.34 & & & & \\
\hline$>=300$ & 0.53 & & & & \\
\hline
\end{tabular}


The experts filled out 18 judgement matrices (i.e. one for the groups, three for the criteria per group, and 14 for the options per criterion). Afterwards, 18 aggregated judgement matrices were computed by geometrically averaging only consistent matrices of experts $(\mathrm{CR} \leq 0.1)$. Appendix $\mathrm{B}$ presents the corresponding priorities and consistency ratio per judgement matrix (for each expert, as well as for the aggregations). Examples are given in Table 7 and Table 8. The final weights were obtained top-down by multiplying the aggregated priorities with the weight of its higher hierarchical layer in Table 6, starting from $100 \%$ (e.g. $0.5214 * 100$ or $52.14 \%$ for the improvement criteria (G2), and $0.1144 * 52.14$ or $5.97 \%$ for the number of business processes (I05)).

Table 7. An example of aggregated judgement matrix with normal priority vector.

\begin{tabular}{|c|c|c|c|c|}
\hline Groups of criteria & G1 & $\mathrm{G} 2$ & G3 & Normal priority vector \\
\hline G1 - Assessment criteria & 1 & 0.55 & 1.86 & 0.3046 \\
\hline $\begin{array}{l}\text { G2 - Improvement criteria } \\
\text { G3 - Non-design criteria }\end{array}$ & & 1 & 2.82 & $\begin{array}{l}0.5214 \\
0.1740\end{array}$ \\
\hline
\end{tabular}

Table 8. An example of aggregated judgement matrix with idealised priority vector.

\begin{tabular}{|l|cccc|}
\hline I05 - Number of business processes & One & More & All & Combination \\
\hline One & 1 & 0.39 & 0.42 & 0.31 \\
More & & 1 & 0.87 & 0.73 \\
All & & & 1 & 0.76 \\
Combination & & & & 1 \\
\hline
\end{tabular}

\begin{tabular}{|c|}
\hline Idealised priority vector \\
\hline 0.3060 \\
0.7285 \\
0.7743 \\
1.0000 \\
\hline
\end{tabular}

$(\mathrm{CR}=0.0015)$

As a rating method, AHP distinguishes normal priority vectors (i.e. with normalised priorities) from idealised priority vectors (i.e. by dividing each normalised priority by the largest one) [23]. Normalised priorities can be summed up to 1 , and are typically used to distribute weights across criteria (Table 7). Idealised priorities express the ideal option per criterion as 1 (Table 8). For instance, the 'number of business processes' (I05) has a weight of 5.97. This weight is assigned to the experts' ideal option (i.e. 'combination'), whereas other options have lower weights. One exception was made for the options within 'presence of capabilities' (I01), for which a normal priority vector is required. Its weight of 17.03 is distributed among all capability areas $(2.69+2.54+2.58+4.47+2.84+1.91)$, instead of assigning 17.03 to the most ideal option (i.e. 'management') and lower weights to the other areas. The latter is impossible as BPMMs cover multiple capability areas. Moreover, weights were assigned to main areas instead of capability clusters to obtain a stricter quality check on the BPMM sample. For instance, the basic cluster can also contain BPMMs that address two of the four basic areas (e.g. only 'optimisation' and 'management') [30]. In our hierarchical model, such BPMMs score lower on this criterion than other BPMMs with all basic areas.

Table 6 also shows that improvement criteria (G2) should be more decisive for BPMM selection than assessment (G1) or non-design (G3) criteria. Ratings are indeed not an end goal, but capability improvements and performance improvements are. The 'presence of capabilities' (I01) has the highest weight as it ultimately represents what is being measured and improved. The 'costs' (N03) received the lowest weight to avoid that an organisation selects a free model that measures the wrong scope of capabilities for that particular organisation (and becomes useless as such). Nonetheless, all criteria of Table 6 reached consensus of being of utmost important for BPMM selection.

Per criterion, a BPMM scores the weight that corresponds to the option to which it applies. For instance, a BPMM that allows measuring one, more and all process(es) in the organisation, scores 5.97 selection points for criterion I05. On the other hand, a BPMM that merely allows measuring separate business processes scores 1.83 selection points. Hence, a BPMM that meets all ideal options reaches the maximum of 100 selection points, whereas a BPMM with the least ideal option on each selected criteria arrives at a selection score of 37.71. As 0 points are assigned for missing values (i.e. criteria absent in the BPMM design documents), a BPMM may theoretically end up with a minimum score of zero. 
Besides a selection score per BPMM, the Delphi study raised the need for a transparency score to indicate how complete the design documents are. In other words, once a BPMM is selected, does the user get sufficient information to start using it? Table 9 extends Table 6 by also reflecting the criteria without consensus. A BPMM scores 1 transparency point for each criterion present in its design documents, -1 per absent criterion and 0 for criteria that are not applicable. Although the Delphi study considered 24 criteria, 3 of them are duplicated for maturity levels and capability levels: 'calculation' (A07-08), 'representation' (A09-10) and 'number of levels' (I06-07). Hence, a transparency score ranges from -27 (i.e. when no criterion is present) to 27 (i.e. when all criteria are present in the BPMM design documents).

\section{Table 9. The transparency requirements of BPMM design documents.}

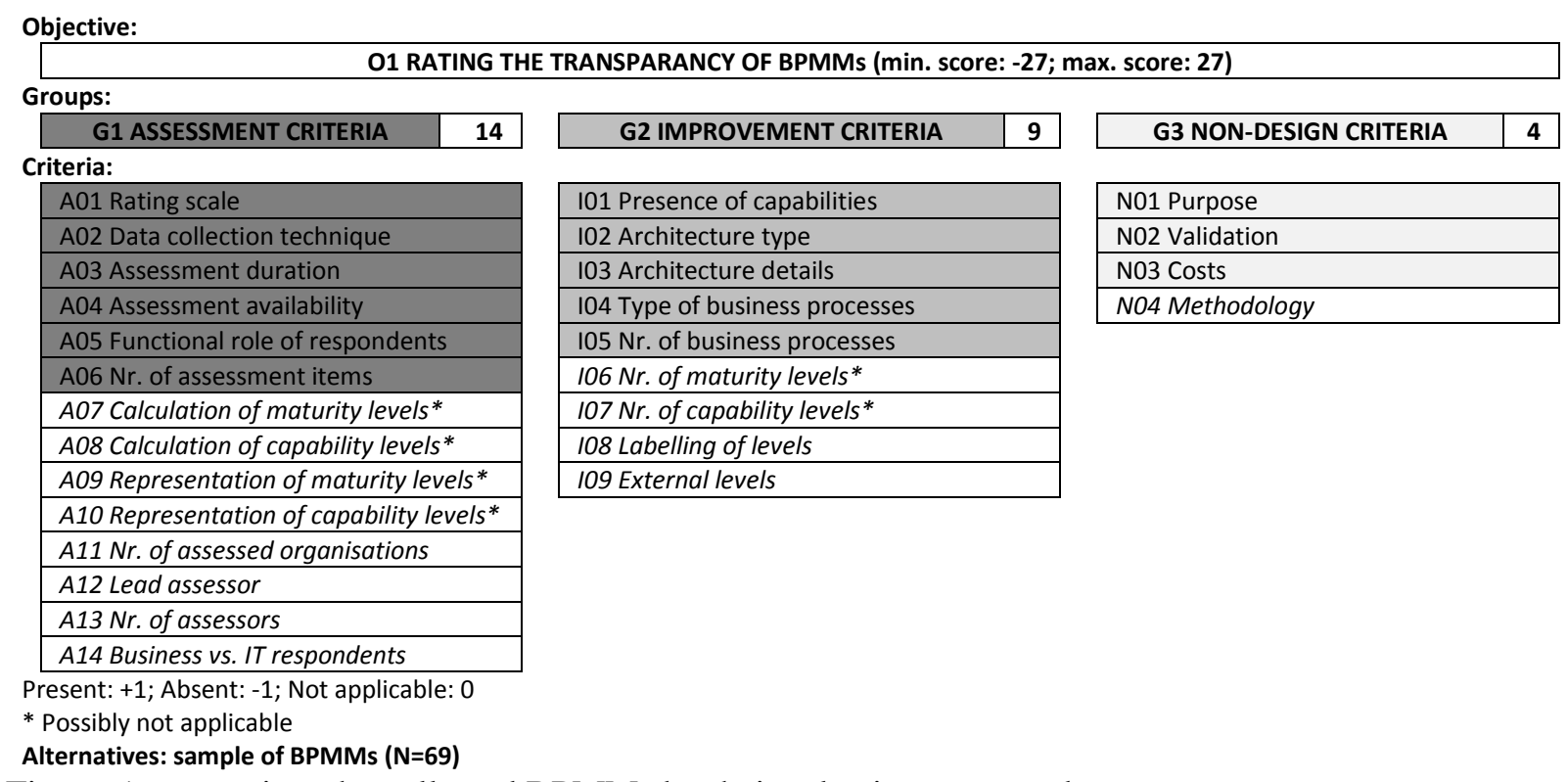

Figure 5 summarises the collected BPMMs by their selection score and transparency score.

\section{(Take in Figure 5)}

Most BPMMs have a selection score between 60 and 80. Only four BPMMs have a higher score, but still below 90 (i.e. MCC1, IDS, BOH, and CGF). 14 BPMMs have a score below 60, of which five below 50 (i.e. RIV, SCH2, AND, STE, ESI2, and SMI). Regarding the transparency score, most BPMMs are situated between 15 and 25. Five models achieved the maximum transparency score of 27 (i.e. SEI, BPT, BIS, IDS and CGF). The two last-mentioned models also have a high selection score, indicating that their design documents are most transparent and mainly contain the ideal options. Figure 5 shows nine outlying BPMMs with a selection score below 60 and a transparency score below five: RIV, SCH2, AND, ESI2, STE, SMI, GAR2, CHA, LEE. They have ten or more missing values on all 27 criteria, and lack half of the selection criteria. As they are less directly usable after selection, we excluded them from the sample to guarantee the quality of the tool's output.

Finally, in a wrap-up round, the Delphi experts were asked to rate their overall satisfaction with the resulting weights ( $1=$ very dissatisfied; $7=$ very satisfied).

\section{(Take in Figure 6)}

With an overall satisfaction rate of $95 \%$ and a mode of score 6 , the vast majority of experts confirmed the resulting ranking and weighing by AHP (Figure 6). 


\subsection{Decision table and questionnaire}

The results were translated into a questionnaire with trade-offs derived from the Delphi comments (Appendix C) and a decision table with the proposed sequence (Appendix D). The online version is called 'BPMM Smart-Selector', and is freely available at a website that we will add after this journal's blind review procedure.

Figure 7 illustrates how the questionnaire appears in the online version. The user can start with those questions that are most relevant to his organisation (and use other questions to refine the results afterwards), or follow the proposed sequence. Question explanations and trade-offs are available in comment fields. If a question, however, seems not relevant for a specific organisation, the user can select "I don't know" to navigate to the next question, or directly click on the title of another question. Multiple answer options can be chosen per question. When options A and B are simultaneously indicated, all BPMMs with A or B are displayed (i.e. OR-combination). For AND-combinations (i.e. $\mathrm{AB})$ a separate option is present, for example, 'Both' in question 5 (e.g. criterion A01).

\section{(Take in Figure 7)}

On the right, a summary is given with the number of matching BPMMs and the responses. We recall from Figure 5 that this matching procedure only considers those BPMMs which passed an initial quality check (i.e. independent of the user's responses). A blocking message is shown when zero BPMMs or one BPMM match the selection. The former is a restriction of the BPMM sample (i.e. not all combinations are addressed in existing BPMMs). Nevertheless, if present, the tool advises to undo the last response, and navigate to the selection table of resulting BPMMs. At any time, the user can switch between the questionnaire and the selection table (Figure 8), or retake the questionnaire.

\section{(Take in Figure 8)}

The selection table shows the responses in green, missing data (i.e. absent in the design documents) in red, and other data in black. If multiple BPMMs match the selection, the user can compare them, refine by answering more questions or request additional information to find the best solution. Concerning the latter, the button "Show/hide additional info" adds the 13 secondary criteria of Table 9. These criteria were not considered decisive for BPMM selection (i.e. outside the questionnaire), but allow a closer look at the proposed BPMMs. Moreover, as multiple BPMMs may theoretically result from the selected criteria, supplementary information could motivate the final choice (as requested by requirement Req2 in Table 1). If no BPMM fits a question to which the user still wants to respond, he is advised to make concessions on less relevant questions and first answer the most relevant questions.

\section{Testing the BPMM decision tool}

An initial version of the BPMM Smart-Selector was tested by employees enrolled for the BPM course of a postgraduate training program. Afterwards, the tool was applied in a multiple-case design that covers the different dimensions of BPMM use. Hence, the number of case studies was determined by the necessity to have illustrative scenarios, being one of the most common evaluation methods within the design-science paradigm [21]. The testers represent organisations of different sizes (i.e. a micro, small, medium and large organisation), whereas the users represent the intended areas of application (i.e. a business scenario for an organisation not yet using a BPMM, a business scenario for an organisation already using a BPMM, and an academic scenario). The business scenarios also represent a profit and a non-profit organisation. Instead of statistical generalisation, we deliberately opted for case design (i.e. characterised by a small set) to illustrate and elaborate on particular contexts of organisations (i.e. why the tool can be used) and their selection process (i.e. how the tool can be used). 
Data saturation was reached, as after pre-testing, the scenarios did not result in significant improvements to the tool.

\subsection{Testing by BPM students}

To test the BPMM Smart-Selector at an early stage, we asked feedback from four BPM freshmen working for organisations of different sizes (i.e. each representing a micro, small, medium and large organisation). This pretest resulted in four improvements: (i) additional comments to clarify the ORand AND-combinations, (ii) a blocking message for zero or one matching BPMM(s), (iii) a button to retake the questionnaire without returning to the homepage, and (iv) the possibility to skip questions. Hence, the criteria are shown in a proposed sequence to guide organisations, but specific organisations still get the opportunity to deviate from this sequence in order to find their best fit.

\subsection{First example: business scenario, not yet using a BPMM}

A first field study was conducted at an international organisation that manufactures chemical products. Due to recent growth, the company is automating its planning activities as a top-down initiative, led by consultants. The new application will replace MS Excel files, and will introduce forecasting. The organisation has a matrix structure, with a horizontal supply chain department crossing functional departments. A BPMM is desired to increase in-house knowledge, and explore opportunities.

The questionnaire was filled out by the European supply chain manager. Most important to him, a BPMM must cover all capabilities (I01) for supply chains (I04). Three BPMMs correspond to this selection: CGF [50], EKN [62], and LMI [46]. All three are free (N03), fully known (A04), and give prescriptive guidance (i.e. implicit or explicit) (I03). CFG was chosen, because it allows benchmarking (N01) and is validated for both its application and its outcomes (N02). The manager is considering its use in all international divisions, and to compare their results with competitors.

\subsection{Second example: business scenario, already using a BPMM}

Another field study took place at the public administration of a large city. The organisation is investing in BPM to obtain work transparency and avoid knowledge losses when the baby boomers retire. In the mid-2000s, the human resource department was extended with a competence centre for organisational change, and five BPM experts started modelling departmental processes. Meanwhile, projects have been launched to reorganise cross-departmental processes. Due to its non-competitive environment, the organisation does not strive for the highest maturity levels. It merely wants to introduce BPM principles into its projects. The organisation already conducted a BPMM assessment once by using WIL [114]. However, its use was stopped because of high charges, and focussing on all processes instead of specific processes and projects.

One of the initial BPM experts used our BPMM Smart-Selector to find a more appropriate BPMM. Most importantly, the BPMM must cover all capabilities (I01), for one or more generic processes (I04) (I05), being fully known (A04), and free (N03). As the two resulting BPMMs, HAM [69] and HAR1 [71], do not allow a quick scan (A06), a concession had to be made on a less relevant criterion. HAM was chosen because it is better validated (N02) and addresses both the maturity of specific processes and all processes in the organisation (I05) (i.e. less assessment items must be answered for only specific processes (A06)). The organisation has decided to use HAM for new projects.

\subsection{Third example: academic scenario}

The BPMM Smart-Selector can also be used by scholars who want to apply a BPMM in their research. For instance, a researcher is investigating why some organisations are not process-oriented (i.e. not striving towards the highest maturity levels) by elaborating on external factors, for example, 
organisation size, sector, market competitiveness, etc. She intended to use the well-known and validated MCC1 [83], but the BPMM Smart-Selector recommends HAM [69].

Her main requirements were that the BPMM must cover all capabilities (I01), presenting maturity levels (I02) for generic processes (I04), being validated for its application and/or its outcomes (N02), fully known (A04) and free (N03). The initial MCC1 satisfies all requirements, except for the capability coverage. Finally, the scholar might consider the proposed BPMM for her research, and recommends the tool to others.

\section{Discussion}

The design-science paradigm follows an iterative approach with build-evaluate cycles. Although it requires years to validate innovative artefacts, this article presents a preliminary evaluation with three common evaluation methods [21]:

- expert evaluation: to assess the criteria of the tool (sections 5.3-5.4);

- prototyping: implementation of the tool to demonstrate the suitability of artefacts (section 5.5);

- in-depth case studies: demonstrations of the tool in real-word situations (section 6).

This section shows to what extent the empirical data collected by the evaluation methods demonstrate our requirements (section 3.1). Delphi and case study data cannot be used for statistical generalisation. Instead, we verify whether requirements Req1-9 are supported by the majority of experts, testers and users with satisfaction rates of 5-6-7 on a 7-point Likert scale, and we evaluate the entire design process in requirement Req10. If this evaluation demonstrates all requirements, it will suggest that our prototype has the potential to offer a well-advised BPMM choice and can be launched.

Table 10. A preliminary evaluation of solution requirements, before the prototype launch.

\begin{tabular}{|c|c|c|c|c|c|c|c|c|}
\hline \multicolumn{2}{|c|}{ Requirement measures } & \multicolumn{2}{|l|}{ Experts } & \multicolumn{2}{|c|}{ Testers } & \multicolumn{2}{|c|}{ Users } & \multirow[t]{2}{*}{ Comment } \\
\hline \multirow[t]{4}{*}{ Req1 } & BPMM before $<>$ after & & & $4 / 4$ & $100 \%$ & $3 / 3$ & $100 \%$ & \\
\hline & $\begin{array}{l}\text { Considering use of BPMM } \\
\text { outcome }\end{array}$ & & & $2 / 4$ & $50 \%$ & $3 / 3$ & $100 \%$ & $\begin{array}{l}\text { Testers without decision } \\
\text { power }\end{array}$ \\
\hline & Satisfied BPMM outcome & & & $2 / 4$ & $50 \%$ & $2 / 3$ & $66.66 \%$ & $\begin{array}{l}\text { One user wants to use the } \\
\text { BPMM before judging utility }\end{array}$ \\
\hline & Recommendations to others & & & $3 / 4$ & $75 \%$ & $2 / 3$ & $66.66 \%$ & Idem \\
\hline Req2 & Single BPMM outcome & & & $4 / 4$ & $100 \%$ & $3 / 3$ & $100 \%$ & \\
\hline Req3 & Satisfied efficiency & & & $4 / 4$ & $100 \%$ & $3 / 3$ & $100 \%$ & 22.71 minutes on average \\
\hline Req4 & Satisfied quality & & & $2 / 4$ & $50 \%$ & $3 / 3$ & $100 \%$ & \\
\hline Req5 & Satisfied expert's criteria & $19 / 20$ & $95 \%$ & & & & & See Figure 4 \\
\hline Req6 & Satisfied weights & $19 / 20$ & $95 \%$ & & & & & See Figure 6 \\
\hline Req7 & Satisfied user's criteria & & & $2 / 4$ & $50 \%$ & $3 / 3$ & $100 \%$ & \\
\hline Req8 & Satisfied question formulations & & & $2 / 4$ & $50 \%$ & $3 / 3$ & $100 \%$ & \\
\hline Req9 & Satisfied sequence & & & $2 / 4$ & $50 \%$ & $3 / 3$ & $100 \%$ & \\
\hline Req10 & Compliance with guidelines & & & & & & & See Table 11 \\
\hline
\end{tabular}

Table 10 recalls that the experts were highly positive about the criteria (Req5) and their weights (Req6). Also the users had positive opinions, particularly regarding the design process (Req7-9), effectiveness (Req2), efficiency (Req3), and quality (Req4). The first requirement needs a refined view as utility is complex to measure in the absence of any alternative tool, and before actually implementing the proposed BPMM. Nevertheless, organisations without an initial BPMM were advised of a BPMM and others were redirected towards a BPMM which better matches their needs (i.e. responses to criteria). Moreover, all three users actually consider using the proposed BPMM. Further on, we note that the metrics of the testers were lower as they refer to the initial version (before making significant tool improvements). Generally, it follows that all metrics concretising requirements Req1-9 were positively evaluated by the majority of experts, testers and users, e.g. with satisfaction rates of 5-6-7 on a 7-point Likert scale. Compliance with the design evaluation guidelines is illustrated in Table 11.

Table 11. The evaluation by IS design guidelines of Hevner et al. [7]. 


\begin{tabular}{l|l}
\hline $\begin{array}{l}\text { 1. Design as an artefact: producing a viable artefact in the form of a construct, a model, a method, or an } \\
\text { instantiation. }\end{array}$ & $\S 3$ \\
\hline 2. Problem relevance: the objective is to develop (technology-based) solutions to important business problems. & $\S 2$ \\
\hline $\begin{array}{l}\text { 3. Design evaluation: the utility, quality, and efficacy of a design artefact must be rigorously demonstrated } \\
\text { through well-executed evaluation methods (e.g. observational, analytical, experimental, testing, or descriptive). }\end{array}$ & $\S 6$ \\
\hline $\begin{array}{l}\text { 4. Research contribution: effective research must provide clear and verifiable contributions regarding the design } \\
\text { artefact itself, its design foundations (i.e. the literature), and/or design methodologies. }\end{array}$ & $\S 8$ \\
\hline 5. Research rigour: applying rigorous methods for both building and testing the design artefact. & $\S 4$ \\
\hline $\begin{array}{l}\text { 6. Design as a search process: the search for an effective artefact requires available means to reach desirable ends } \\
\text { while satisfying laws in the problem environment. }\end{array}$ & $\S 4.1$ \\
\hline $\begin{array}{l}\text { 7. Communication of research: effectively presenting to technology-oriented and management-oriented } \\
\text { audiences. }\end{array}$ & $\begin{array}{l}\text { Conference \& journal } \\
\text { papers }\end{array}$ \\
\hline
\end{tabular}

The search process of guideline 6 involves iteratively identifying decision criteria and developing alternative tools. As explained in section 4.1, a content analysis with open coding was first conducted to identify BPMM design characteristics. They were supplemented by peer feedback before starting the Delphi study. Regarding alternative tools, we considered a mandatory and optional order of questions by using MS Excel or a website. Furthermore, guideline 7 is met by presenting preliminary findings at conferences on the design-science paradigm (Desrist) and on information systems (Confenis) and synthesising the research in this article.

Finally, as the information is judged consistent with the design requirements, our prototype can be launched to proceed with large-scale studies. We hereby recommend limiting the measures for the utility requirement to a comparison between the intended BPMM before and after using the tool, supplemented by a consideration of actual use.

\section{Research contributions and limitations}

This article conforms to the design-science paradigm by demonstrating how a decision tool (BPMM Smart-Selector) can solve an important problem, i.e. how organisations can choose the right BPMM to start improving business processes and enhance performance. The hypotheses or solution requirements are derived from decision-making theories. The tool is innovative, as any selection support for maturity models was still lacking. The solution construction or methodology is transparent, grounded in the literature, and with an iterative solution process. Solution instantiations are used to demonstrate the tool and its managerial implications. Regarding the theoretical implications, the design artefacts are our main contribution, including their instantiation in the BPMM Smart-Selector. The study extends the BPMM literature by offering insight into the decision criteria, their trade-offs and weights. The latter allow a critical view on existing BPMMs by calculating scores. Furthermore, the warm-up discussions about business process capabilities give more evidence for the usefulness of the clustering of Van Looy et al. [30]. As the clusters were experienced quite naturally by almost the entire Delphi expert panel, our findings corroborate their previous work [30] by subjectmatter experts. Finally, a design process for a BPMM decision tool is proposed by addressing the design theory components of Walls et al. [33], which also involves a proposed quality check for BPMMs.

In our research, the following methodological limitations are taken into account.

- Expert panel. The study typically relies on a small, non-random sample. Hence, careful attention was given to expert selection based on role and region. We used strict consensus conditions (i.e. four in total) to ensure a broad empirical basis for the selected criteria.

- Codification panel. The typical emphasis on impersonal communication might cause an interpretation bias. Therefore, responses were analysed by multiple coders (including an independent coder from another university), and the experts received feedback per round.

- Delphi design. The initial criteria might bias the first round. Nevertheless, experts could give open comments at any time and propose an unlimited list of missing criteria. This semi-structured approach also allowed common ground among experts. 
- Hierarchical model. Future research could consider a supplementary AHP calculation within the BPMM Smart-Selector. As the hierarchical model assumes independent criteria, AHP could be extended to the analytical network process (ANP) to include real-time feedback [23]. For instance, an organisation can value guidance higher than costs, but when choosing between BPMMs with good guidance, costs become more important. Currently, feedback is limited to a selection table (Figure 8).

- BPMM sample. The BPMM Smart-Selector is a prototype tool implementation, based on BPMM design documents of 2010 or earlier. Information available in other or more recent documents is not taken into account. These BPMM details can be easily corrected in the database. The sample is thus a way to test the decision tool, and can be updated or extended with additional BPMMs afterwards. Nevertheless, to our knowledge, its large size of 69 BPMMs is more comprehensive than other BPMM studies.

- Testing. Testing was limited to illustrate the use of our BPMM Smart-Selector. We do not statistically evaluate a scale or relationships between variables. The latter could be done in followup research to investigate which organisation type chooses which cluster (I01). Hence, making the BPMM Smart-Selector available at this time is thought to be beneficial to organisations and researchers. Further feedback will be used to prepare a next release.

Notwithstanding these limitations, our study was rigorously executed. Its soundness is now evaluated by qualitative research criteria [13].

- Credibility (i.e. internal validity). Accurate judgements were guaranteed by the absence of group pressures in a Delphi study, involving international academics and practitioners interested in BPMM selection, coding triangulation by multiple coders, feedback reports checked by the experts, and high response rates.

- Transferability (i.e. external validity). The BPMM Smart-Selector can be extended to other BPMMs or decision criteria without changing the database structure. Future research could investigate whether our methodology can be generalised to other decision tools (e.g. for selecting maturity models on business-IT alignment, project management, etc.).

- Dependability (i.e. reliability). Hasson et al. [6] assert that Delphi results cannot be repeated due to the small sample. Therefore, experts were carefully selected based on their role and region, and their responses were analysed by multiple coders. Moreover, the testing phase did not indicate missing decision criteria.

- Confirmability (i.e. objectivity). The initial criteria were corroborated by peer feedback. Objective conditions were defined to select experts and reach consensus. The coders first individually coded the responses before consolidating in team. Furthermore, the experts could rate and comment the overall importance per round, and received objective feedback reports. Finally, the tool was successfully tested.

\section{Conclusion}

Choosing a BPMM for what you want to achieve is critical (i.e. fit for purpose). Therefore, an online decision tool, called BPMM Smart-Selector, was built and tested. As illustrated, it serves organisations and academics wishing to choose a BPMM. The tool consists of a questionnaire with 14 decision criteria and trade-offs, linked to a decision table that guides users to the best matching BPMM. Particularly, it concerns six assessment criteria, five improvement criteria, and three nondesign criteria. They were elicited after a content analysis of 69 BPMMs and an international Delphi study (or consensus-seeking decision-making). One of the criteria (i.e. presence of capabilities) corroborates the findings of Van Looy et al. [30] by confirming three clusters of business process capabilities addressed by BPMMs. Additionally, AHP (or multi-criteria decision-making) was used to 
weigh criteria. The final scores for selection and transparency allowed a thorough BPMM overview and an a priori quality check to decide whether a BPMM is included in the tool.

We genuinely applied the IS design guidelines of Hevner et al. [7], the IS artefact types of March and Smith [15], and the IS design theory components of Walls et al. [33]. The design requirements are demonstrated by the empirical evidence that we collected, and can be reused for large-scale studies. Future research could investigate whether our methodology allows theory building on other decision tools (e.g. for selecting other maturity models). Another avenue is to build a theory to explain why organisations choose for a specific capability cluster, based on the data collected by the tool.

\section{Acknowledgements}

We truly thank the coders and the expert panel for their continuing participation throughout the different Delphi rounds. Furthermore, we thank the testers for using our prototype tool.

\section{References}

[1] W. Afzal, D. Roland, M.N. Al-Squri, Information asymmetry and product valuation: an exploratory study. Journal of Information Science 35(2) (2009) 192-203.

[2] V.A. Banuls, J.L. Salmeron, Foresighting key areas in the information technology industry. Technovation 28 (2008) 103-111.

[3] N. Dalkey, O. Helmer, An experimental application of the Delphi method to the use of experts. Management Science 9(3) (1963) 458-467.

[4] W.M. Goldstein, Judgements of relative importance in decision making. Organizational Behavior and Human Decision Processes 47 (1990) 313-336.

[5] C.M. Harvey, R.J. Koubek, Cognitive, social, and environmental attributes of distributed engineering collaboration. Human Factors and Ergonomics in Manufacturing 10(4) (2000) 369-393.

[6] F. Hasson, S. Keeney, H. McKenna, Research guidelines for the Delphi survey technique. Journal of Advanced Nursing 32(4) (2000) 1008-1015.

[7] A.R. Hevner, S.T. March, J. Park, S. Ram, Design science in information systems research. MIS Quarterly 28(1) (2004) 75-105.

[8] C.-C. Hsu, B.A. Sandford, Minimizing non-response in the Delphi process: how to respond to non-response. Practical Assessment, Research \& Evaluation 12(17) (2007) 1-6.

[9] J.J. Jiang et al., An exploration on the relationship between software development process maturity and project performance. Information \& Management 41 (2004) 279-288.

[10] R.L. Keeney, H. Raiffa, Decisions with multiple objectives. preferences and value tradeoffs, Cambridge University Press, Cambridge, 1993.

[11] G. Kou, Y. Shi, S. Wang, Multiple criteria decision making and decision support systems. Decision Support Systems 51 (2011) 247-249.

[12] M.C. Lacity, M.A. Janson, Understanding qualitative data: a framework of text analysis methods. Journal of Management Information Systems 11(2) (1994) 137-155.

[13] Y.S. Lincoln, E.G. Guba, Naturalistic inquiry, Sage Publications, California, 1985.

[14] A.M. Maier, J. Moultrie, J.P. Clarkson, Assessing organizational capabilities: reviewing and guiding the development of maturity grids. IEEE-TEM 59(1) (2012) 138-159.

[15] S.T. March, G.F. Smith, Design and natural science research on information technology. Decision Support Systems 15(4) (1995) 251-266.

[16] T. Mettler, A design science research perspective on maturity models in information systems. Institute of Information Management, Report (2009) BE IWI/HNE/03.

[17] T. Mettler, P. Rohner, Situational maturity models as instrumental artifacts for organizational design, DESRIST Proceedings (2009) 1-9.

[18] H. Mintzberg, Managerial work. Management Science 18(2) (1971) B97-B110. 
[19] P.M. Mullen, Delphi: myths and reality. Journal of Health Organization and Management 17(1) (2003) 37-52.

[20] C. Okoli, S.D. Pawlowski, The Delphi method as a research tool: an example, design constructions and applications. Information \& Management 42 (2004) 15-29.

[21] K. Peffers, M. Rothenberger, T. Tuunanen, R. Vaezi, Design science research evaluation, in: K. Peffers, M. Rothenberger, B. Kuechler (Eds.), Design science research in information systems, Springer, Berlin-Heidelberg, 2012, pp. 398-410.

[22] M. Röglinger, J. Pöppelbuss, J. Becker, Maturity models in business process management. Business Process Management Journal 18(2) (2012) 7-7.

[23] R.W. Saaty, Decision making in complex environments, 2003, retrieved September 2011, from: http://creativedecisions.net

[24] T.L. Saaty, An exposition of the AHP in reply to the paper "Remarks on the analytical hierarchy process". Management Science 36(3) (1990) 259-268.

[25] S.A. Sheard, Evolution of the frameworks quagmire, IEEE Computer Society 34 (2001) 96-98.

[26] H.A. Simon, Rational decision making in business organizations. The American Economic Review 69(4) (1979) 493-513.

[27] M. Taleai, A. Mansourian, Using Delphi-AHP method to survey major factors causing urban plan implementation failure. Journal of Applied Sciences 8(15) (2008) 2746-2751.

[28] A.H. Van De Ven, A.L. Delbecq, The effectiveness of nominal, Delphi, and interacting group decision making processes. The Academy of Management Journal 17(4) (1974) 605-621.

[29] A. Van Looy, M. De Backer, G. Poels, Defining business process maturity. A journey towards excellence. Total Quality Management \& Business Excellence 22(11) (2011) 1119-1137.

[30] A. Van Looy, M. De Backer, G. Poels, A conceptual framework and classification of capability areas for business process maturity. Enterprise Information Systems (2013) (in press, online version available at $i$ First).

[31] J. Vanthienen, G. Wets, Creating integration of the decision table formalism with a relational datatbase environment. Information Systems, 20(7) (1995) 595-616.

[32] J. vom Brocke, M. Rosemann, Foreword, in: J. vom Brocke, M. Rosemann (Eds.), Handbook on Business Process Management 2, Springer, Berlin Heidelberg, 2010, pp. vii-ix.

[33] J.G. Walls, G.R. Widmeyer, O.A. El Sawy, Assessing information system design theory in perspective. Journal of Information Technology Theory and Application, 6(2) (2004) 43-58.

[34] M. Wang, D. Vogel, W. Ran, Creating a performance-oriented e-learning environment: a design science approach. Information \& Management 48 (2011) 260-269.

[35] M. Weske, Business Process Management, Springer, Berlin, 2007.

\section{Additional references to the collected BPMMs (N=69)}

[36] AberdeenGroup, Global supply chain benchmark report, 2006, retrieved June 23, 2010, from: https://www-935.ibm.com/services/us/igs/pdf/aberdeen-benchmark-report.pdf

[37] D.L. Anderson, H.L. Lee, The Internet-enabled supply chain, in: D.L. Anderson (Ed.), Achieving supply chain excellence through technology, Montgomery Research, 2000, pp. 15-20.

[38] G. Aouad, et al., A synchronised process/IT model to support the co-maturation of processes and IT in the construction sector. Time Research Institute, CIB report (1998) Salford.

[39] APQC, Supply-chain council SCOR-mark survey, 2007, retrieved April 23, 2010, from: http://www.apqc.org/scc

[40] C. Armistead, S. Machin, Implications of business process management for operations management, International Journal of Operations \& Production Management 17 (1997) 886-898.

[41] G. Aryee, M.M. Naim, C. Lalwani, Supply chain integration using a maturity scale. Journal of Manufacturing Technology Management 19(5) (2008) 559-575. 
[42] J.B. Ayers, Supply chain maturity self-assessment, 2010a, retrieved June 23, 2010, from: http://ayers-consulting.com/SC\%20Maturity\%20Self\%20Assessment.htm

[43] J.B. Ayers, Supply chain project management, Taylor and Francis Group, Boca Raton, 2010b.

[44] Bisnez Management, Business process management onderzoek, 2010, retrieved June 20, 2010, from: http://www.bisnez.org/

[45] T. Böhme, Supply chain integration, The University of Waikato, Waikato, New Zealand, 2008.

[46] L.M. Boone, A.J. Colaianni, J.R. Hardison, et al., The GAIA supply chain sustainability maturity model, 2009, retrieved June 23, 2010, from: http://www.lmi.org/logistics/logistics.aspx

[47] BPMInstitute, BPM market assessment survey, 2010, retrieved June 20, 2010, from: http://2010stateofbpm.surveyconsole.com/

[48] BPT Group, Welcome to 8 Omega, 2008, retrieved June 20, 2010, from: http://bptg.seniordev.co.uk/8omega.aspx

[49] J. Campbell, J. Sankaran, An inductive framework for enhancing supply chain integration. International Journal of Production Research 43(16) (2005) 3321-3351.

[50] CGF, The global scorecard, 2010, retrieved June 23, 2010, from: http://www.globalscorecard.net/ [51] B. Champlin, Dimensions of business process change, 2008, retrieved June 20, 2010, from: https://www.bpminstitute.org/uploads/media/Champlin-6-25-08.pdf

[52] Chicago Consulting, Supply chain maturity: a self-assessment, 2010, retrieved June 23, 2010, from: http://www.chicago-consulting.com/

[53] D.J. Closs, D.A. Mollenkopf, A global supply chain framework. Industrial Marketing Management 33 (2004) 37-44.

[54] S. Cohen, J. Roussel, Strategic supply chain management, McGraw-Hill, New York, 2005.

[55] T. de Bruin, M. Rosemann, Using the delphi technique to identify BPM capability areas, ACIS Proceedings (2007) 642-653.

[56] Deloitte, Het business maturity model, 2010, retrieved June 21, 2010, from: http://www.deloitte.com/view/nl_NL/nl/diensten/consulting/

[57] I.M. de Soria, J. Alonso, L. Orue-Echevarria, M. Vergara, Developing an enterprise collaboration maturity model, ICE Proceedings (2009) 1-8.

[58] I. DeToro, T. McCabe, How to stay flexible and elude fads. Quality Progress (3) (1997) 55-60.

[59] P. Dowdle, J. Stevens, The process audit and PBM roadmap. A PBM Program Perspective, 2007, retrieved June 21, 2010, from: http://www.cam-i.org/

[60] P. Dowdle, J. Stevens, B. McCarty, D. Daly, The process-based management loop. The Journal of Corporate Accounting \& Finance (2005) 55-60.

[61] T. Dwyer, BPMInstitute's state of business process management, 2006, retrieved June 20, 2010, from: http://www.bpminstitute.org/

[62] eKNOWtion, Supply chain maturity monitor, 2009, retrieved June 23, 2010, from: http://www.eknowtion.com/

[63] T. Enkawa, Logistics Scorecard (LSC) in Japan, 2005, retrieved June 23, 2010, from: http://transportal.fi/Hankkeet/eglo/.383.pdf

[64] FAA, FAA-iCMM, 2001, retrieved March 15, 2010, from: http://www.faa.gov/

[65] FAA, FAA-iCMM Appraisal Method, 2006, retrieved March 15, 2010, from: http://www.faa.gov/

[66] D.M. Fisher, The business process maturity model. BPTrends September (2004) 1-4.

[67] P. Fraser, C. Farrukh, M. Gregory, Managing product development collaboration: a process maturity approach. Journal of Engineering Manufacture 217(11) (2003) 1499-1519.

[68] R.A. Gardner, The process-focused organization, ASQ, Quality Press, Milwaukee, 2004.

[69] M. Hammer, The process audit, Harvard Business Review 4 (2007) 111-123. 
[70] P. Harmon, Evaluating an organization's business process maturity. BPTrends March (2004) 111.

[71] H. J. Harrington, Process Management Excellence, Paton Press, California, 2006.

[72] J. Holmes, Building supply chain communities, 1997, retrieved April 24, 2010, from: http://criticalcomputing.com/

[73] IBM, The IBM global business services Mainland China value chain study, 2007, retrieved February June 23, 2010, from: http://www-935.ibm.com/services/us/gbs/thoughtleadership/\#Next

[74] IDS Scheer, BPM Maturity Check, 2010, retrieved June 22, 2010, from: http://www.bpmmaturity.com/

[75] ISO/IEC, Part 2: performing an assessment, ISO/IEC, Geneva, 2003.

[76] ISO/IEC, Part 7: assessment of organizational maturity, ISO/IEC, Geneva, 2008.

[77] J. Lee, D. Lee, S. Kang, vPMM: a value based Process Maturity Model, in: R. Lee, G. Hu, H. Miao (Eds.), Computer and Information Science, Springer, Berlin Heidelberg, 2009, pp. 193-213.

[78] F. Luyckx, IDS BPM roadmap assessment, 2007, retrieved June 22, 2010, from: http://www.idsscheer.nl/set/2238/Overview\%20BPM\%20maturity\%20model.pdf

[79] A.M. Magdaleno et al., Towards collaboration maturity in business processes: an exploratory study in oil production processes. Information Systems Management 25 (2008) 302-318.

[80] R.S. Maull, D.R. Tranfield, W. Maull, Factors characterising the maturity of BPR programmes. International Journal of Operations \& Production Management 48 (2003) 596-624.

[81] K. McCormack, Supply chain management maturity, in: K. McCormack (Ed.), Business process maturity, Booksurge Publishing South Carolina, 2007, pp. 73-103.

[82] K. McCormack, W.C. Johnson, Business Process Orientation: Gaining the e-Business Competitive Advantage, St. Lucie Press, Florida, 2001.

[83] T. McLaren, A measurement model for web-enabled supply chain integration, Bled Proceedings (2006) paper 18.

[84] M.J. Melenovsky, J. Sinur, BPM maturity model identifies six phases for successful BPM adoption, Gartner Research, Stamford, 2006.

[85] T.H. Netland, E. Alfnes, H. Fauske, How mature is your supply chain? Supply Chain Maturity Assessment Test, EurOMA Proceedings (2007) 1-10.

[86] OMG, Business Process Maturity Model, 2008, retrieved December 2, 2009, from: http://www.omg.org/

[87] Oracle, BPM lifecycle assessment, 2008a, retrieved June 23, 2010, from: http://bpmready.nvishweb.com/

[88] Oracle, State of the business process management market 2008, 2008b, retrieved May 4, 2010, from: http://www.oracle.com/

[89] E. Ostolaza, A.B. Garcia, EFQM/SPICE integrated model, PROFES Proceedings (1999) 437452.

[90] C.C. Poirier, F.J. Quinn, M.L. Swink, Diagnosing greatness, J. Ross Publishing, Fort Lauderdale, 2010.

[91] J.-P. Pritchard, C. Armistead, Business process management: lessons from European business. Business Process Management Journal 5(1) (1999) 10-32.

[92] N. Ramasubbu, M.S. Krishnan, Leveraging global resources: a process maturity framework for managing distributed software product development, AMCIS Proceedings (2005) 3085-3090.

[93] G. Remoreras, Achieving the highest level of process culture maturity, 2009, retrieved June 1, 2010, from: http://mysimpleprocesses.com

[94] J. Riverola, A general approach to the case gathering phase, 2001, retrieved July 6, 2010, from: http://catalcatel.iese.edu/WEBSCM/Archivos/MaturityModel.pdf

[95] M. Rohloff, Process management maturity assessment, AMCIS Proceedings (2009) paper 631. 
[96] M. Rosemann, T. de Bruin, Towards a business process management maturity model, ECIS Proceedings (2005) paper 37.

[97] Rummler-Brache Group, Business process management in U.S. firms today, 2004, retrieved June 23, 2010, from: http://rummler-brache.com/

[98] M. Scavillo, Business process transformation in the software industry, 2008, retrieved June 23, 2010, from: http://www.sdn.sap.com/irj/scn/

[99] A.-W. Scheer, BPM = business process management = business performance management, 2007, retrieved April 27, 2010, from: http://www.professor-scheer-bpm.com/

[100] T.I. Schoenfeldt, A practical application of supply chain management principles, ASQ, Milwaukee, 2008.

[101] SEI, CMMI for development, 2006, retrieved December 3, 2009, from: http://www.sei.cmu.edu/ [102] SEI, CMMI for acquisition, 2007, retrieved February 11, 2010, from: http://www.sei.cmu.edu/

[103] SEI, CMMI for services, 2009, retrieved February 11, 2010, from: http://www.sei.cmu.edu/

[104] T.M., Simatupang, R. Sridharan, The collaboration index. International Journal of Physical Distribution \& Logistics Management 35(1) (2005) 44-62.

[105] R. Skrinjar, V. Bosilj-Vuksic, M.I. Stemberger, The impact of business process orientation on financial and non-financial performance, Business Process Management Journal 14 (2008) 738-754.

[106] H. Smith, P. Fingar, Process management maturity models. BPTrends July (2004).

[107] A. Spanyi, Towards process competence, 2004, retrieved April 6, 2010, from: http://www.spanyi.com/images/BPM\%20Towards.pdf

[108] G.C. Stevens, Integrating the supply chain. International Journal of Physical Distribution \& Logistics Management 19(8) (1989) 3-8.

[109] Supply-Chain Council, Supply-chain operations reference-model, 2008, retrieved June 14, 2010, from: http://supply-chain.org/about/scor

[110] R.S. Tapia, M. Daneva, P.van Eck, R. Wieringa, Towards a business-IT alignment maturity model for collaborative networked organizations, EDOC Workshop Proceedings (2008) 276-287.

[111] J. Tolsma, D. de Wit, Effectief procesmanagement, Eburon, Delft, 2009.

[112] J. van den Bergh, Supply chain maturity scan, 2010, retrieved June 23, 2010, from: http://www.jvdbconsulting.com/

[113] VICS, Collaborative planning, forecasting and replenishment (CPFR), 2004, retrieved June 14, 2010, from: http://www.vics.org/

[114] P. Willaert, J. Van den Bergh, J. Willems, D. Deschoolmeester, The process-oriented organisation: a holistic view, BPM Proceedings (2007) 1-15.

[115] P.M. Wognum, E.C. Faber, Infrastructures for collaboration in virtual organisations. International Journal of Networking and Virtual Organisations 1(1) (2002) 32-54.

\section{Appendix A. Collected BPMMs (N=69)}

\begin{tabular}{|c|c|c|c|}
\hline ID & Author(s) & \multicolumn{2}{|l|}{ BPMM name } \\
\hline \multicolumn{4}{|c|}{ (1) Business process } \\
\hline \multicolumn{4}{|c|}{ (1.1) Academic } \\
\hline $\mathrm{AOU}$ & Aouad et al. & Co-maturation model for synchronising $\mathrm{BP}$ and IT & [38] \\
\hline ARM & Armistead, Machin and Pritchard & BPM's degree of progress (part of a survey) & {$[40 ; 91]$} \\
\hline DET & DeToro and McCabe & Process condition rating model & {$[58]$} \\
\hline HAM & Hammer & Process and Enterprise Maturity Model & [69] \\
\hline HAR 1 & Harrington & Process maturity grid & [71] \\
\hline LEE & Lee, Lee and Kang & Value-based process maturity model (vPMM) & [77] \\
\hline MAU & Maull, Tranfield and Maull & BPR maturity model & {$[80]$} \\
\hline MCC1 & McCormack and Johnson & BPO maturity model & {$[82]$} \\
\hline $\mathrm{ROH}$ & Rohloff & Process management maturity assessment & [95] \\
\hline ROS & Rosemann, de Bruin and Power & BPM maturity model & {$[55 ; 96]$} \\
\hline SEI & SEI (Carnegie Mellon University) & $\begin{array}{l}\text { - Capability maturity model integration (CMMI) } \\
\text { - CMMI appraisal method (SCAMPI) }\end{array}$ & {$[101 ; 102 ; 103]$} \\
\hline SKR & Skrinjar et al. & BPO maturity model & {$[105]$} \\
\hline
\end{tabular}




\begin{tabular}{|c|c|c|c|}
\hline ID & Author(s) & BPMM name & ence(s) \\
\hline WIL & Willaert et al. & Holistic BPO maturity framework & {$[114]$} \\
\hline \multicolumn{4}{|c|}{ (1.2) Non-academic } \\
\hline BIS & Bisnez Management & $\begin{array}{l}\text { BPM maturity model (in Dutch: 'BPM } \\
\text { volwassenheidsmodel') }\end{array}$ & [44] \\
\hline BPM & BPMInstitute & State of BPM (part of a survey) & {$[47,61]$} \\
\hline BPT & $\begin{array}{l}\text { BP Transformations Group and BPGroup } \\
\text { (former BPM Group) }\end{array}$ & $\begin{array}{l}8 \text { Omega ORCA (Organisational readiness and } \\
\text { capability assessment) }\end{array}$ & {$[48]$} \\
\hline CAM1 & CAM-I & Process-based management loop & {$[60]$} \\
\hline CAM2 & CAM-I & $\begin{array}{l}\text { Process-based management assessment and } \\
\text { implementation road map }\end{array}$ & [59] \\
\hline $\mathrm{CHA}$ & Champlin (ABPMP) & Process management maturity model & {$[51]$} \\
\hline DEL & Deloitte and Utrecht University & Business maturity model and scan & [56] \\
\hline ESI1 & ESI, European Software Institute & EFQM/SPICE integrated model & [89] \\
\hline FAA & FAA, Federal Aviation Administration & $\begin{array}{l}\text { - FAA integrated capability maturity model } \\
\text { - FAA-iCMM appraisal method (FAM) }\end{array}$ & {$[64 ; 65]$} \\
\hline FIS & Fisher (BearingPoint) & Business process maturity model & {$[66]$} \\
\hline GAR1 & Gardner & Process improvement road map & {$[68]$} \\
\hline GAR2 & Gartner & BPM maturity and adoption model & [84] \\
\hline HAR2 & Harmon (BPTrends) & Informal BP maturity evaluation model & {$[70]$} \\
\hline IDS & IDS Scheer, Software AG & $\begin{array}{l}\text { - BPM maturity check } \\
\text { - BPM road map assessment }\end{array}$ & {$[74 ; 78]$} \\
\hline ISO & ISO/IEC & ISO/IEC 15504 & {$[75 ; 76]$} \\
\hline O\&I & O\&i & BPM scan & [111] \\
\hline OMG & OMG & Business process maturity model (BPMM) & {$[86]$} \\
\hline ORA & Oracle and BEA Systems & BPM lifecycle assessment survey & {$[87 ; 88]$} \\
\hline REM & Remoreras & Process culture maturity model & [93] \\
\hline RUM & Rummler-Brache Group & Process Performance Index & [97] \\
\hline SAP & SAP & Process maturity analysis and plan & {$[98]$} \\
\hline SCH1 & Scheer & BPM check-up & [99] \\
\hline SMI & Smith and Fingar & Process management maturity model & {$[106]$} \\
\hline SPA & Spanyi & BP competence grid & {$[107]$} \\
\hline \multicolumn{4}{|c|}{ (2) Supply chain } \\
\hline \multicolumn{4}{|c|}{ (2.1) Academic } \\
\hline ARY & Aryee, Naim and Lalwani & SC integration maturity model & [41] \\
\hline $\mathrm{BOH}$ & Böhme and Childerhouse & SC integration evaluation tool/maturity model & [45] \\
\hline CAM3 & Campbell and Sankaran & SC integration enhancement framework & [49] \\
\hline MCC2 & McCormack & SC management maturity model & {$[81]$} \\
\hline MCL & McLaren & SC integration measurement model & {$[83]$} \\
\hline MIC & Michigan State University & $21^{\text {st }}$ Century Logistics Framework & {$[53]$} \\
\hline NET & Netland, Alfnes and Fauske & SC maturity assessment test (SCMAT) & {$[85]$} \\
\hline RIV & Riverola & SC management/Technology maturity model & [94] \\
\hline TOK & Tokyo Institute of Technology & Logistics scorecard (LSC) & [63] \\
\hline \multicolumn{4}{|c|}{ (2.2) Non-academic } \\
\hline $\mathrm{ABE}$ & AberdeenGroup & Global SC maturity framework & [36] \\
\hline AND & Andersen Consulting (Accenture) & SC continuum & [37] \\
\hline CGF & $\begin{array}{l}\text { CGF, Consumer Goods Forum (former } \\
\text { GCI) }\end{array}$ & $\begin{array}{l}\text { Global scorecard for efficient consumer response } \\
\text { capability }\end{array}$ & {$[50]$} \\
\hline CGR & CGR Management Consulting & SC management maturity model & {$[42 ; 43]$} \\
\hline $\mathrm{CHI}$ & Chicago Consulting & SC maturity model & {$[52]$} \\
\hline $\mathrm{CSC}$ & $\begin{array}{l}\text { CSC, SCM Review Magazine, Michigan } \\
\text { State University }\end{array}$ & $\begin{array}{l}\text { - SC maturity model (until 2006) } \\
\text { - Ten SC competencies (as from 2007) }\end{array}$ & [90] \\
\hline EKN & eKNOWtion & $\mathrm{SC}$ maturity monitor $\left(\mathrm{SCM}^{2}\right)$ & {$[62]$} \\
\hline IBM & IBM & SC maturity model & [73] \\
\hline JER & $\begin{array}{l}\text { Jeroen van den Bergh Consulting and VU } \\
\text { University Amsterdam }\end{array}$ & SC maturity scan & [112] \\
\hline LMI & LMI Research Institute & GAIA SC sustainability maturity model & [46] \\
\hline MAN & Manugistics and JDA Software & SC Compass & [72] \\
\hline PMG & PMG and PRTM & SC maturity model & [54] \\
\hline $\mathrm{SCC}$ & SCC, Supply Chain Council and APQC & $\begin{array}{l}\text { SCORmark Survey (for benchmarking, resulting in } \\
\text { an improvement road map) }\end{array}$ & [39;109] \\
\hline $\mathrm{SCH} 2$ & Schoenfeldt & SC mgt maturity model & {$[100]$} \\
\hline STE & Stevens & SC integration model & {$[108]$} \\
\hline \multicolumn{4}{|c|}{ (3) Collaboration } \\
\hline \multicolumn{4}{|c|}{ (3.1) Academic } \\
\hline FRA & Fraser, Farrukh and Gregory & $\begin{array}{l}\text { Collaboration maturity grid (for new product } \\
\text { introduction and development) }\end{array}$ & [67] \\
\hline MAG & Magdaleno et al. & Collaboration maturity model (ColabMM) & [79] \\
\hline RAM & Ramasubbu and Krishnan & $\begin{array}{l}\text { Process maturity framework for managing distributed } \\
\text { software product development }\end{array}$ & [92] \\
\hline SIM & Simatupang and Sridharan & SC Collaboration index & [104] \\
\hline TAP & Tapia et al. & IT-enabled collaborative networked organisations & {$[110]$} \\
\hline
\end{tabular}




\begin{tabular}{lllll}
\hline ID & \multicolumn{1}{c}{ Author(s) } & \multicolumn{1}{c}{ BPMM name } & Reference(s) \\
\hline \multirow{2}{*}{ WOG } & Wognum and Faber & maturity model (ICoNOs MM) & & \\
& & $\begin{array}{l}\text { Fast reactive extended enterprise } \\
\text { assessment framework (FREE-CAF) }\end{array}$ & capability & {$[115]$} \\
\hline $\mathbf{( 3 . 2 )}$ Non-academic & & & & \\
\hline ESI2 & ESI, European Software Institute & Enterprise Collaboration Maturity Model & [57] & {$[113]$} \\
VIC & VICS & CPFR rollout readiness self-assessment & \\
\hline
\end{tabular}




\section{Appendix B. Results of AHP}

\begin{tabular}{|c|c|c|c|c|c|c|c|c|c|c|c|c|c|c|c|c|c|c|}
\hline & R01 & R03 & R04 & R05 & R06 & R07 & R10 & R11 & R12 & R13 & R14 & R15 & R16 & R18 & R19 & R21 & R22 & Final \\
\hline O1* & 0.0000 & 0.0000 & 0.0000 & 0.0000 & 0.0000 & 0.0370 & $\underline{0.1304}$ & 0.0279 & 0.0000 & 0.0624 & 0.0000 & 0.0370 & 0.2939 & 0.0624 & 0.0000 & 0.0136 & 0.0516 & 0.0035 \\
\hline G1 & 3333 & 0.2000 & 0.4286 & 0.4737 & 0.1667 & 0.1047 & $\overline{0.2808}$ & 0.3420 & 0.2000 & 0.6491 & 0.3000 & 0.1047 & $\overline{0.2099}$ & 0.2789 & 0.4000 & 0.3332 & 0.2493 & 0.3046 \\
\hline G2 & 0.3333 & 0.6000 & 0.4286 & 0.4737 & 0.6667 & 0.2583 & 0.5842 & 0.5769 & 0.6000 & 0.2790 & 0.6000 & 0.2583 & 0.7297 & 0.6491 & 0.4000 & .5917 & .5936 & 0.5214 \\
\hline G3 & 3333 & 0.2000 & 0.1429 & 0.0526 & 0.1667 & 6370 & 0.1350 & 0.0811 & 2000 & 0.0719 & 0.1000 & 0.6370 & .0604 & 0.0719 & 0.2000 & 0.0751 & 0.1571 & 0.1740 \\
\hline G1* & .0039 & 0.0000 & 0.0678 & 0.1411 & 0.0418 & 0.0339 & 0.1088 & 0.0315 & 0.0152 & 0.0469 & 0.0431 & 0.0186 & 0.0240 & 0.0250 & 0.0358 & 0.0358 & 0.0680 & 0.0019 \\
\hline A01 & 1378 & 0.1000 & 0.2568 & $\overline{0.1051}$ & 0.4054 & 0.1137 & $\overline{0.0800}$ & 0.3961 & 0.1049 & 0.3587 & 0.4203 & 0.1521 & 0.2845 & 0.2382 & 0.2365 & 0.0392 & 0.4064 & 0.2554 \\
\hline A 02 & 1284 & 0.1000 & 0.4243 & 0.1694 & 0.1867 & 0.4329 & 0.1513 & 0.3961 & 0.1049 & 0.3158 & 0.2693 & 0.1047 & .3448 & 0.1052 & 0.2365 & 0.1991 & .0657 & 0.2443 \\
\hline A03 & 0.3394 & 0.2000 & 0.0908 & 0.6047 & 0.0371 & 0.1537 & 0.4419 & 0.0676 & 0.2916 & 0.0585 & 0.0773 & 0.3824 & 0.0346 & 0.0442 & 0.0457 & 0.0801 & 0.1909 & 0.1282 \\
\hline A04 & 0.1284 & 0.1000 & 0.1442 & 0.0354 & 0.1306 & 0.0560 & 0.2536 & 0.0336 & 0.1692 & 0.0281 & 0.0497 & 0.2564 & 0.1637 & 0.0442 & 0.2365 & 0.1671 & 0.1090 & 0.1255 \\
\hline A05 & 0.1284 & 0.1000 & 0.0260 & 0.0263 & 0.1867 & 0.2039 & 0.0299 & 0.0445 & 0.2916 & 0.1854 & 0.0263 & 0.0666 & 0.1044 & 0.1052 & 0.1547 & 0.3153 & 0.0371 & 0.1253 \\
\hline A06 & 0.1378 & 0.4000 & 0.0580 & 0.0590 & 0.0535 & 0.0398 & 0.0432 & 0.0621 & 0.0377 & 0.0535 & 0.1571 & 0.0378 & 0.0681 & 0.4628 & 0.0901 & 0.1991 & 0.1909 & 0.1213 \\
\hline G2* & 0297 & 0.0461 & 0.0434 & 0.0131 & 0.0478 & 0.0000 & 0.0700 & $\underline{0.1392}$ & 0.0351 & 0.0159 & 0.0167 & 0.0786 & 0.0088 & 0.0260 & 0.0131 & 0.0491 & 0.0217 & 0.0002 \\
\hline I01 & 0.1529 & 0.1665 & 0.3200 & 0.2482 & 0.4677 & 0.2000 & 0.4699 & $\overline{0.4885}$ & 0.0919 & 0.2838 & 0.4702 & 0.4872 & 0.2805 & 0.4847 & 0.2576 & 0.4661 & 0.1073 & 0.3267 \\
\hline I02 & 0.1529 & 0.3375 & 0.0829 & 0.1458 & 0.0628 & 0.2000 & 0.1441 & 0.0690 & 0.1987 & 0.2838 & 0.1211 & 0.2620 & 0.2805 & 0.2202 & 0.2576 & 0.0323 & 0.3580 & 0.2024 \\
\hline I03 & 0.4302 & 0.3375 & 0.1151 & 0.1097 & 0.0877 & 0.2000 & 0.0792 & 0.1221 & 0.1987 & 0.2838 & 0.0902 & 0.1384 & 668 & 0.2202 & 0.2576 & & 0.2981 & 0.1971 \\
\hline I04 & 0.1250 & 0.0536 & 0.4361 & 0.2482 & 0.2206 & 0.2000 & 0.0448 & 0.2827 & 0.4720 & 0.1132 & 0.2678 & 0.0726 & 0.0695 & 0.0375 & 0.1513 & 0.2235 & 0.0685 & 0.1594 \\
\hline I05 & 0.1389 & 0.1049 & 0.0459 & 0.2482 & 0.1611 & 0.2000 & 0.2619 & 0.0377 & 0.0388 & 0.0354 & 0.0506 & 0.0399 & 0.1027 & 0.0375 & 0.0759 & 0.2459 & 0.1681 & 0.1144 \\
\hline G3* & 0000 & 0.0089 & 0.0824 & 0.3617 & 0.0824 & 0.0000 & 0.0707 & 0.0279 & 0.0000 & 0.0000 & 0.0176 & 0.0370 & 237 & 0.0370 & 0.0000 & 0.0280 & 0.1304 & 0.0006 \\
\hline N01 & 0.1250 & 0.2970 & 0.2255 & $\overline{0.7520}$ & 0.6267 & 0.6000 & 0.1172 & 0.5770 & 0.3333 & 0.3333 & 0.6250 & 0.6370 & 0.1998 & 0.6370 & 0.4000 & 0.6586 & $\overline{0.0972}$ & 0.4258 \\
\hline N02 & .7500 & 0.5396 & 0.6738 & $\theta .1966$ & 0.2797 & 0.2000 & 0.2684 & 0.3420 & 0.3 & 0.3333 & 0.2385 & 0.1047 & 0.6833 & 0.2583 & 0.4000 & 0.1852 & 0.7007 & 0.3774 \\
\hline $\mathrm{N} 03$ & 0.1250 & 0.1634 & 0.1007 & 0.0514 & 0.0936 & 0.2000 & 0.6144 & 0.0811 & 0.3333 & 0.3333 & 0.1365 & 0.2583 & 0.1169 & 0.1047 & 0.2000 & 0.1562 & 0.2021 & 0.1968 \\
\hline A01 & 0.0053 & - & 0.0904 & 0.0000 & 0.0824 & 0.0000 & 0.1464 & 0.1897 & 0.0237 & 0.0000 & 0.0176 & 0.0370 & .0006 & 0.1126 & 0.0000 & 0.5411 & 0.0000 & 0.0017 \\
\hline Quali & 2154 & 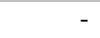 & 0.1228 & 1.0000 & 63 & 0.3333 & 0.1357 & 0.2589 & 24 & 0.3333 & 1.0000 & 0.1644 & 615 & 1.0000 & 1.0000 & 0.0534 & 00 & 0.4592 \\
\hline Quant & 0.4642 & - & 1.0000 & 0.5000 & 0.1494 & 0.3333 & 0.3684 & 0.0805 & 0.1710 & 0.3333 & 0.2184 & 0.4055 & 0.1156 & 0.0790 & 1.0000 & 0.2311 & 0.2000 & 0.3833 \\
\hline Both & 1.0000 & - & 0.4524 & 0.5000 & 1.0000 & 1.0000 & 1.0000 & 1.0000 & 1.0000 & 1.0000 & 0.5724 & 1.0000 & 1.0000 & 0.2811 & 1.0000 & 1.0000 & 1.0000 & 1.0000 \\
\hline A02 & 0.0000 & 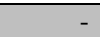 & 0.0824 & 0.0000 & 0.0904 & 0.0000 & 0.0707 & 0.0311 & 0.0000 & $\underline{0.2836}$ & 0.0516 & 0.0370 & 0.0000 & 0.0772 & 0.0000 & $\underline{0.5411}$ & $\underline{0.4196}$ & 0.0019 \\
\hline Obj & 1.0000 & - & 0.4463 & 1.0000 & 0.1228 & 0.2000 & 0.1908 & 0.3989 & 0.2000 & $\overline{0.3420}$ & 1.0000 & 0.4055 & 1.0000 & 0.4421 & 1.0000 & 0.2311 & 0.2732 & 0.4807 \\
\hline Subj & & - & 0.1494 & & & & & & & 0.0835 & 0.3150 & & & 0.0838 & 1.0000 & 0.0534 & 0.0747 & 0.3062 \\
\hline Both & .0000 & - & 1.0000 & 1.0000 & 1.0000 & 1.0000 & 1.0000 & 1.0000 & 1.0000 & 1.0000 & 0.3969 & 1.0000 & 1.0000 & 1.0000 & 1.0000 & 1.0000 & 1.0000 & 1.0000 \\
\hline $\mathrm{A} 03$ & 0.0063 & - & 0.2090 & 0.4189 & 0.2837 & 0.0000 & 0.2090 & 0.0904 & 0.0516 & 0.0000 & 0.0147 & 0.0068 & 0.0012 & $\underline{0.1126}$ & 0.1304 & 0.5411 & 0.0516 & 0.0090 \\
\hline Day & 020 & $x_{1}$ & 0.3969 & 1.0000 & 0.0836 & 1.00 & 0.3969 & 0.0921 & & 10000 & 0.8740 & & & 0.0790 & 0.1387 & 0.0534 & 1.0000 & 0.4176 \\
\hline Week & 1.0000 & 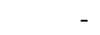 & 1.0000 & 0.2125 & 1.0000 & 1.0000 & 1.0000 & 1.0000 & 1.0000 & 1.0000 & 1.0000 & 1.0000 & & 1.0000 & 1.0000 & 0.2311 & 0.6300 & 1.0000 \\
\hline Long & 0.275 & 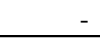 & 0.1050 & 0.0581 & 0.3420 & 0.3333 & 0.0900 & 0.3393 & & 0.3333 & 0.3817 & 0.1314 & 1.0000 & 0.2811 & 0.4808 & 1.0000 & 0.1587 & 0.3611 \\
\hline A04 & 0.0516 & - & 0.0311 & 0.0000 & $\underline{0.1758}$ & 0.0000 & $\underline{0.2593}$ & 0.0960 & 0.0516 & 0.0000 & 0.0000 & $\underline{0.1126}$ & 0.0000 & 0.0772 & $\underline{0.1304}$ & $\underline{0.5411}$ & $\underline{0.1304}$ & 0.0060 \\
\hline Know & 0.6300 & 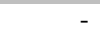 & 1.0000 & 1.0000 & 1.0000 & 1.0000 & 1.0000 & 0.1043 & & 1.0000 & 0.4999 & 1.0000 & 00 & 1.0000 & 1.0000 & 1.0000 & 1.0000 & 1.0000 \\
\hline Partial & & - & 0.2992 & & & & 02705 & & & & 1.0000 & 0.2811 & & & & & & 0.8659 \\
\hline Un & 0.1184 & - & 0.1194 & 1.0000 & 0.0935 & 0.2000 & 0.0665 & 0.4566 & 0.1323 & 1.0000 & 0.2500 & 0.0790 & 0.1667 & 0.0838 & 0.1387 & 0.0534 & 0.1387 & 0.3335 \\
\hline A05 & 0.0000 & - & 0.0000 & 0.0000 & 0.0000 & 0.0000 & 0.0000 & 0.0000 & 0.0000 & 0.0000 & 0.0000 & 0.0000 & 0.0000 & 0.0000 & 0.0000 & 0.0000 & 0.0000 & 0.0000 \\
\hline Int & 0.20 & 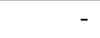 & 1.000 & 1.00 & 0 & 1.00 & 3333 & 0.2500 & 0.3333 & 1.0000 & 0.3333 & 1.0000 & 0.2000 & 0.1429 & 1.0000 & 1.0000 & 1.0000 & 0.5500 \\
\hline Ext & 1.0000 & - & 0.2500 & 1.0000 & 1.0000 & 1.0000 & 1.0000 & 1.0000 & 1.0000 & 1.0000 & 1.0000 & 1.0000 & 1.0000 & 1.0000 & 1.0000 & 1.0000 & 1.0000 & 1.0000 \\
\hline A06 & & & & 0.0019 & & & $0.0 / 68$ & 0.0677 & & 55 & 0.0520 & 0.0506 & $\overline{04}$ & 0.0956 & $\underline{0.1199}$ & 0.2085 & $\underline{0.2975}$ & 0.0094 \\
\hline $0-19$ & 0.8034 & - & 0.4604 & & & & 0.6369 & & & & 0.1786 & & & 0.3351 & $\overline{0.2491}$ & $\overline{0.4716}$ & 1.0000 & 0.4494 \\
\hline 49 & 1.0000 & 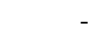 & 1.0000 & 0.7856 & 0.3921 & 1.0000 & 1.0000 & 0.7105 & 0.6727 & 1.0000 & 0.2952 & 1.0000 & 0.6741 & 1.0000 & 1.0000 & 1.0000 & 1.0000 & 1.0000 \\
\hline 99 & 0.6325 & - & 0.6724 & 0.1198 & 0.7622 & 1.0000 & 0.2707 & 1.0000 & 1.0000 & 1.0000 & 1.0000 & 0.5320 & 1.0000 & 0.5508 & 1.0000 & 0.4856 & 0.3745 & 0.8410 \\
\hline
\end{tabular}




\begin{tabular}{|c|c|c|c|c|c|c|c|c|c|c|c|c|c|c|c|c|c|c|}
\hline & R01 & R03 & R04 & R05 & R06 & R07 & R10 & R11 & R12 & R13 & R14 & R15 & R16 & R18 & R19 & R21 & R22 & Final \\
\hline 299 & 0.3846 & 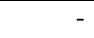 & 0.1391 & 0.1198 & 1.0000 & 1.0000 & 0.1247 & 0.3204 & 0.2174 & 0.1869 & 0.5249 & 0.1777 & 1.0000 & 0.0611 & 0.5578 & 0.1298 & 0.1378 & 0.3632 \\
\hline$>=300$ & 0.2380 & - & 0.0712 & 0.1198 & 0.0982 & 0.1249 & 0.0670 & 0.0869 & 0.0794 & 0.0967 & 0.1168 & 0.0900 & 0.5093 & 0.0611 & 0.1237 & 0.0470 & 0.0523 & 0.1448 \\
\hline I01* & 0.0153 & 0.0144 & 0.0362 & 0.0044 & 0.0305 & 0.0000 & 0.0153 & 0.0387 & 0.0029 & 0.0353 & 0.0166 & 0.0327 & $\underline{0.1940}$ & 0.0000 & 0.0226 & 0.0396 & 0.0144 & 0.0008 \\
\hline Model & 0.1257 & 0.0764 & 0.0646 & 0.1089 & 0.1495 & 0.1667 & 0.3154 & 0.1041 & 0.1485 & 0.3178 & 0.1055 & 0.1663 & 0.0214 & 0.1667 & 0.0398 & 0.2210 & 0.2282 & 0.1577 \\
\hline Depl & 0.1188 & 0.0588 & 0.0314 & 0.1019 & 0.3573 & 0.1667 & 0.2430 & 0.3790 & 0.0817 & 0.3178 & 0.0888 & 0.1153 & 0.0559 & 0.1667 & 0.0932 & 0.1109 & .1080 & 0.1490 \\
\hline Optim & 0.0575 & 0.1067 & 0.0826 & 0.1019 & 0.1125 & 0.1667 & 0.1730 & 0.2543 & 0.1485 & 0.1793 & 0.1676 & 0.3340 & 0.0262 & 0.1667 & 0.1061 & 0.1037 & .0747 & 0.1515 \\
\hline Mgt & 0.2230 & 0.3883 & 0.3822 & 0.2516 & 0.2501 & 0.1667 & 1201 & 0.1817 & 0.2698 & 1006 & 0.3652 & 0.2570 & 0.3989 & 1667 & 0.2536 & 0.2719 & 2283 & 0.2624 \\
\hline Cult & 0.2230 & 0.2356 & 0.1817 & 0.2178 & 0.0849 & 0.1667 & 0.0845 & 0.0317 & 0.2698 & 0.0452 & 0.2011 & 0.0726 & 0.3664 & 0.1667 & 0.2536 & 0.2719 & .2283 & 0.1670 \\
\hline Struct & 0.2520 & 0.1342 & 0.2574 & 0.2178 & 0.0457 & 0.1667 & 0.0640 & 0.0491 & 0.0817 & 0.0393 & 0.0719 & 0.0547 & 0.1311 & 0.1667 & 0.2536 & 0.0207 & 0.1325 & 0.1124 \\
\hline I02 & 0.0000 & - & 0.0089 & 0.0147 & 0.0516 & 0.0370 & $\underline{0.1304}$ & $\underline{0.1570}$ & 0.0370 & $\underline{0.1304}$ & 0.0147 & 0.0370 & 0.0033 & 0.0624 & $\underline{0.1304}$ & .0000 & 0516 & 0.0000 \\
\hline Cont & 0.5000 & - & 0.5503 & 1.0000 & 0.1587 & 0.1644 & $\overline{0.4808}$ & $\overline{1.0000}$ & 0.4055 & $\overline{0.4807}$ & 0.3817 & 0.4055 & 0.9285 & 0.4297 & $\overline{0.2314}$ & 0.3333 & .6300 & 0.6896 \\
\hline Stage & 0.5000 & - & 0.3059 & 0.3815 & 0.2520 & 1.0000 & 0.2311 & 0.2988 & 0.1644 & 1.0000 & 1.0000 & 1.0000 & 0.2155 & 108 & 1.0000 & 000 & 000 & 0.7104 \\
\hline Both & 1.0000 & - & 1.0000 & 0.4369 & 1.0000 & 0.4055 & 1.0000 & $\theta .1116$ & 1.0000 & 0.0994 & 0.8740 & 0.1644 & 1.0000 & 1.0000 & 0.4807 & 0.3333 & 0.3969 & 1.0000 \\
\hline $\mathrm{I03}$ & 0.0000 & - & 0.0036 & 0.0516 & $\underline{0.1190}$ & 0.0000 & $\underline{0.1304}$ & 0.0624 & $\underline{0.2090}$ & 0.0772 & 0.0707 & 0.0068 & 0.0904 & 0.0624 & 0.0000 & 0.0000 & $\underline{0.1350}$ & 0.0074 \\
\hline Descr & 0.5000 & - & 0.1882 & 1.0000 & 0.1007 & 1.0000 & 1.0000 & 0.2579 & 0.3969 & 1.0000 & 1.0000 & 0.1314 & 0.1228 & 0.1108 & 0.2000 & 1.0000 & 0.0991 & 0.5771 \\
\hline Im pr & 1.0000 & - & 1.0000 & 0.6300 & 0.2838 & 1.0000 & 0.4808 & 1.0000 & 0.1575 & 0.1895 & 0.4368 & 0.3625 & 1.0000 & 1.0000 & 1.0000 & 0.3333 & .4808 & 1.0000 \\
\hline Ex pr & 0.2500 & - & 0.3542 & 0.3969 & 1.0000 & 1.0000 & 0.2311 & 0.1108 & 1.0000 & 0.0838 & 0.1908 & 1.0000 & 0.2715 & 0.4297 & 1.0000 & 1.0000 & 1.0000 & 0.5685 \\
\hline I04 & 0.0000 & - & 0.0000 & 0.0000 & 0.0000 & 0.0000 & 0.0000 & 0.0000 & 0.0000 & 0.0000 & 0.0000 & 0.0000 & 0.0000 & 0.0000 & 0.0000 & 0.0000 & 0.0000 & 0.0000 \\
\hline Gener & 1.0000 & 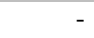 & 1.0000 & 1.0000 & 1.0000 & 10000 & 1.0000 & 0.1429 & 0.2500 & 1.0000 & 0.4999 & 1.0000 & 1.0000 & & 1.0000 & 1.0000 & 1.0000 & 1.0000 \\
\hline Dom & 0.3333 & - & 0.3333 & 0.5000 & 0.3333 & 0.3333 & 0.1667 & 1.0000 & 1.0000 & 0.2000 & 1.0000 & 1.0000 & 0.2000 & 0.2000 & 0.2000 & 0.4999 & 1.0000 & 0.5348 \\
\hline I05 & 0.0732 & - & 0.0183 & 0.0000 & 0.0592 & 0.0016 & 0.0227 & 0.0495 & 0.0297 & 0.0579 & 0.0116 & 0.0286 & 0.0116 & 0.0163 & 0.0579 & 0.0934 & 0.0000 & 0.0015 \\
\hline One & 0.0791 & 15 & 0.1445 & 0.5000 & 0.1340 & 0.3493 & 0.1419 & 0.1784 & 0.0899 & 0.0840 & 0.3426 & 0.1136 & 0.2420 & & 0.1113 & 000 & . 0000 & 0.3060 \\
\hline More & 0.2201 & - & 0.2778 & 1.0000 & 0.1889 & 0.1913 & 0.6048 & 1.0000 & 0.2064 & 1.0000 & 1.0000 & 1.0000 & 0.4080 & 0.1706 & 0.4584 & 1.0000 & 1.0000 & 0.7285 \\
\hline All & 0.2201 & 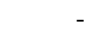 & 0.6743 & 0.5000 & 0.3859 & 1.0000 & 0.9999 & & & & & & & & 999 & & 000 & 0.7743 \\
\hline Comb & 1.0000 & - & 1.0000 & 0.5000 & 1.0000 & 1.0000 & 1.0000 & 0.5173 & 1.0000 & 4277 & .5931 & 0.2584 & 1.0000 & 458 & 1.0000 & 0.1831 & 1.0000 & 1.0000 \\
\hline N01 & 0.0516 & - & 0.0176 & 0.0209 & $\underline{0.1758}$ & 0.0370 & $\underline{0.1304}$ & 0.0734 & 0.0000 & 0.1126 & 0.0036 & 0.0000 & 0.0237 & $\underline{0.2836}$ & 0.0000 & $\underline{0.1037}$ & 0.0000 & 0.0017 \\
\hline Aware & 0.4200 & 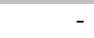 & 0.5724 & 1.0000 & $\overline{1.0000}$ & & $\overline{1.0000}$ & 0.1084 & 1.0000 & 1.0000 & 1.0000 & 1.0000 & 1.0000 & $\overline{1.0000}$ & 1.0000 & $\overline{1.0000}$ & 1.0000 & 0.9689 \\
\hline Bench & 1.0000 & - & 1.0000 & 0.5793 & 0.3057 & 0.4055 & 0.1156 & 1.0000 & 1.0000 & 0.2811 & 0.5313 & 1.0000 & 0.2924 & 0.3420 & 1.0000 & 0.3467 & 1.0000 & 1.0000 \\
\hline Certif & 0.1323 & - & 0.2184 & 0.0959 & 0.0935 & 0.1644 & 0.4807 & 0.3293 & 0.3333 & 0.0790 & 0.1882 & 1.0000 & 0.1710 & 0.0650 & 0.3333 & 0.1202 & 0.2000 & 0.3140 \\
\hline N02 & 0.00 & & & & & & & & & & & 000 & & & & & 000 & \\
\hline Applic & 0.2500 & - & 0.2500 & 1.0000 & 0.2000 & & & 0.4999 & 0.2500 & & & 1.0000 & 0.1250 & 29 & 0.2000 & 0.2000 & 0.5000 & 0.3100 \\
\hline Outc & 1.0000 & - & 1.0000 & 1.0000 & 1.0000 & 1.0000 & 1.0000 & 1.0000 & 1.0000 & 1.0000 & 1.0000 & 1.0000 & 1.0000 & 1.0000 & 1.0000 & 1.0000 & 1.0000 & 1.0000 \\
\hline N03 & 0.0000 & 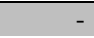 & 0.0000 & 0.0000 & 0.0000 & 0.0000 & $\overline{0000}$ & 0.0000 & 0.0000 & 0.0000 & 0.0000 & 0.0000 & 0.0000 & 0.0000 & 0.0000 & 0.0000 & 0.0000 & 0.0000 \\
\hline Free & 0.5000 & - & 1.0000 & 1.0000 & 1.0000 & 1.00 & 1.0000 & 0.4999 & 1.0000 & 1.0000 & 1.0000 & 1.0000 & 0.2000 & 1.0000 & 1.0000 & 1.0000 & 1.0000 & 1.0000 \\
\hline Charg & 1.0000 & - & 0.2000 & 0.5000 & 0.3333 & 0.2000 & 0.1429 & 1.0000 & 0.3333 & 0.1111 & 1.0000 & 1.0000 & 1.0000 & 0.1111 & 0.3333 & 0.4999 & 0.1111 & 0.4310 \\
\hline
\end{tabular}

Gre $=$ consioncy ratio $(\mathrm{CR} \leq 0.1)$

The other comparisons use idealised priority vectors, obtained by dividing each normal priority vector entry by the largest normal priority vector entry. 


\section{Appendix C. Questionnaire with final criteria and trade-offs}

\begin{tabular}{|c|c|}
\hline I01 & 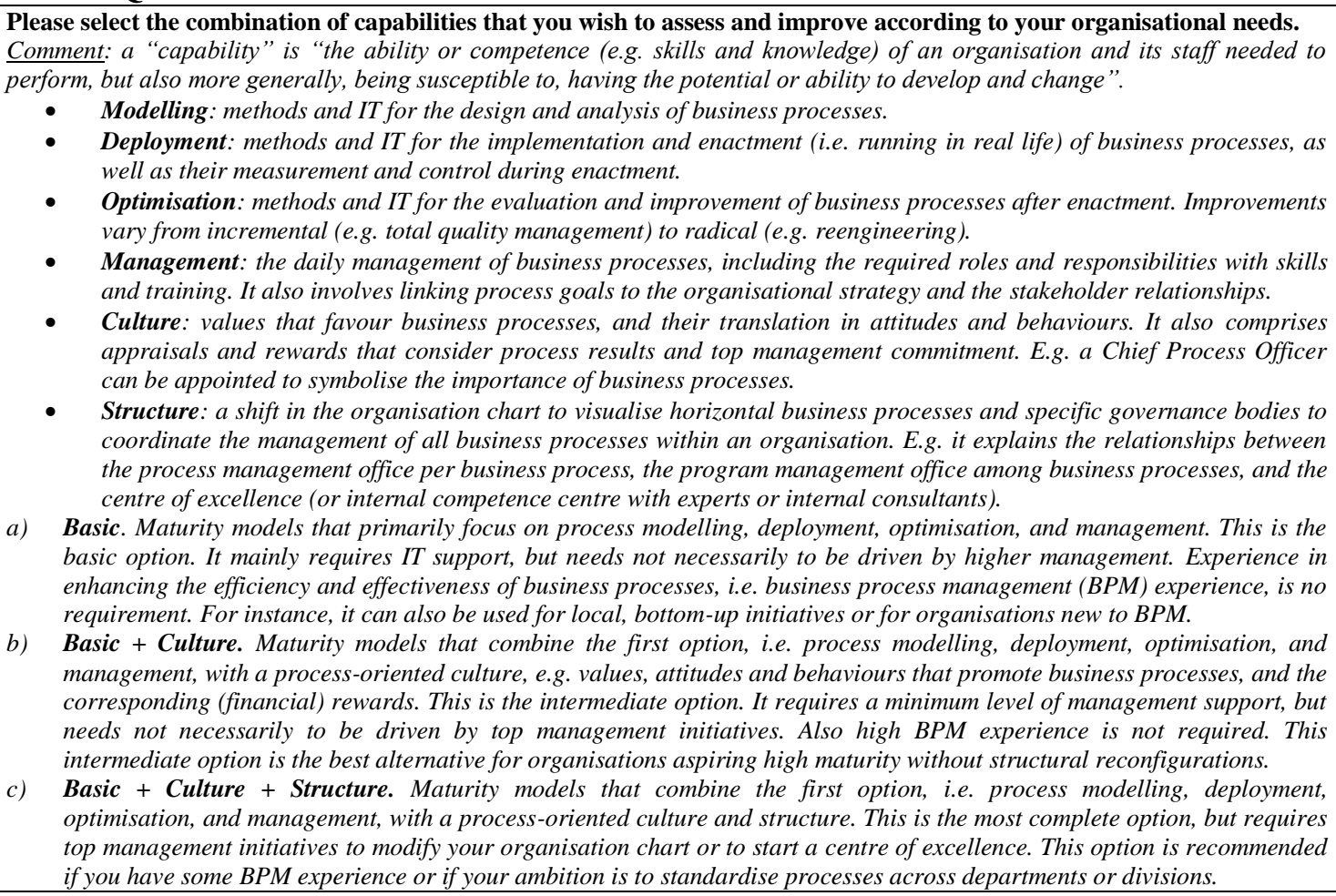 \\
\hline I02 & $\begin{array}{l}\text { Must the maturity model define a road map per capability (continuous) and/or a road map for overall maturity (staged)? } \\
\text { Comment: it concerns linking (maturity or capability) levels to capabilities in a step-by-step plan, which explains how to reach } \\
\text { each consecutive level. The road map can be used to gradually guide (generic, not personalised) improvements. } \\
\text { a) Only continuous: capability levels exist and are linked to each capability. This means that multiple road maps are } \\
\text { presented, i.e. one per capability, which explain how to reach each consecutive capability level. Organisations can assess } \\
\text { and improve each capability separately, and thus decide to improve capabilities at a different pace or limit their scope to } \\
\text { only those capabilities they are interested in. } \\
\text { Only staged: maturity levels exist and are linked to all capabilities. This means that one road map is presented for overall } \\
\text { maturity, i.e. for all capabilities together, which explains how to reach each consecutive maturity level. All capabilities are } \\
\text { simultaneously assessed and improved. The emphasis is on overall advancements, instead of individual capabilities. } \\
\text { c) Both: maturity models which are simultaneously continuous and staged. }\end{array}$ \\
\hline I03 & $\begin{array}{l}\text { How much guidance must the maturity model give on your journey towards higher maturity? } \\
\text { Comment: it concerns the extent to which the road map (i.e. step-by-step plan) explains which criteria (i.e. goals and best } \\
\text { practices) must be satisfied before reaching each particular level. } \\
\text { a) Descriptive: the road map is limited to a high-level description, without defining criteria per level. As it gives less support, } \\
\text { it is suited for organisations wishing to become acquainted with maturity models, or for organisations which are highly } \\
\text { experienced with business process improvements } \\
\text { Implicit prescriptive: the road map has criteria interwoven in the assessment questions, i.e. with an ordinal scale or a } \\
\text { matrix, that explain all capabilities per level. Assessors can derive the criteria from the assessment questions } \\
\text { c) Explicit prescriptive: the road map lists criteria separately from the assessment questions }\end{array}$ \\
\hline I04 & $\begin{array}{l}\text { Must the maturity model be generic (i.e. for business processes in general) or domain-specific (e.g. for business processes } \\
\text { in supply chains or collaboration situations)? } \\
\text { a) Generic, i.e. for business processes in general instead of adapted to particular business domains. The terminology, e.g. in } \\
\text { the assessment questions, is likely to be more holistic, which makes benchmarking easier across business domains. } \\
\text { b) Domain-specific for supply chains } \\
\text { c) Domain-specific for collaboration situations }\end{array}$ \\
\hline A01 & $\begin{array}{l}\text { Which type of data must be collected during an assessment? } \\
\text { a) Only qualitative data: with open questions or with nominal or ordinal rating scales. E.g.: descriptions or the level of } \\
\text { agreement to a statement. These rating scales provide in-depth descriptions, which allow delving into details and thus } \\
\text { identifying bias. However, they depend on the skills of the assessors. } \\
\text { Only quantitative data: with discrete, interval or ratio rating scales. E.g.: process performance measures or business } \\
\text { results. These rating scales can be easily statistically analysed and compared, independent of the assessors' interpretations. } \\
\text { c) Both: maturity models which simultaneously collect qualitative and quantitative data. }\end{array}$ \\
\hline A02 & $\begin{array}{l}\text { How must information be collected during an assessment? } \\
\text { a) Only objectively: by document reviews of existing, written material, e.g. websites, annual reports, policies, standards, } \\
\text { business plans and documents, process models, process performance reports, job descriptions of process owners, historical } \\
\text { records, etc. They give an idea of how focal organisations work, without interrupting individuals or activities. Such } \\
\text { objective evidence can minimise biased results by (particularly internal) assessors and respondents. } \\
\text { Only subjectively: by questionnaires, interviews, focus groups, observations, etc. They gather information about how the } \\
\text { focal organisations actually work, and frequently allow clarifying responses. Such personal beliefs can only minimise } \\
\text { biased results if some precautions are taken, e.g., (1) if the assessment is lead by a third party (i.e. an external, independent } \\
\text { person), (2) if multiple assessors and respondents are involved, (3) if the data collection technique and terminology of }\end{array}$ \\
\hline
\end{tabular}




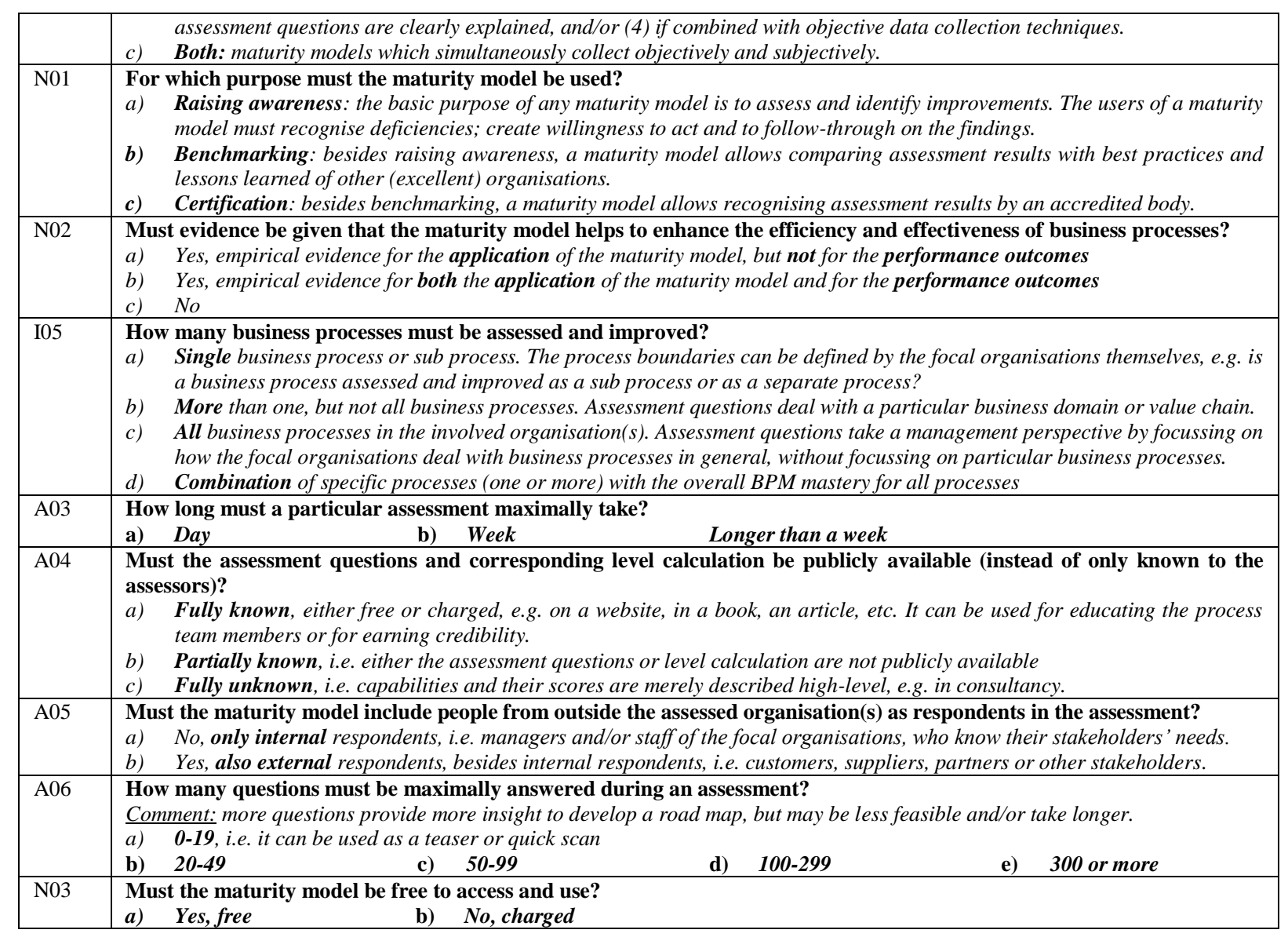




\section{Appendix D. Decision tables with the proposed sequence of questions}

For cluster A: basic capabilities

\begin{tabular}{|c|c|c|c|c|c|c|c|c|c|c|c|c|}
\hline$\overline{\mathrm{I} 01}$ & \multicolumn{12}{|c|}{ Cluster A: basic capabilities } \\
\hline $\mathrm{I} 02$ & \multicolumn{4}{|c|}{ Continuous } & \multicolumn{8}{|c|}{ Staged } \\
\hline $\mathrm{I} 03$ & \multicolumn{2}{|c|}{ Descr } & Impl prescr & Expl prescr & \multirow{2}{*}{\multicolumn{4}{|c|}{$\begin{array}{c}\text { Descr } \\
\text { Generic }\end{array}$}} & \multirow{2}{*}{\multicolumn{3}{|c|}{$\frac{\text { Impl prescr }}{\text { Generic }}$}} & Expl prescr \\
\hline $\mathrm{I} 04$ & Generic & $\mathrm{SC}$ & $\mathrm{SC}$ & $\mathrm{SC}$ & & & & & & & & Generic \\
\hline $\mathrm{A} 01$ & Quali & Both & Quanti & Quali & Quali & \multirow{2}{*}{\multicolumn{3}{|c|}{ Both }} & \multicolumn{3}{|c|}{ Quali } & Quali \\
\hline $\mathrm{A} 02$ & Subj & Subj & Obj & Subj & Both & & & Subj & \multicolumn{3}{|c|}{ Subj } & Subj \\
\hline N01 & Aware & Aware & $\begin{array}{c}\text { Aware or } \\
\text { Bench }\end{array}$ & $\begin{array}{c}\text { Aware or } \\
\text { Bench }\end{array}$ & Aware & \multicolumn{2}{|c|}{ Aware } & Bench & \multicolumn{3}{|c|}{ Aware } & Aware \\
\hline N02 & Applic & Outc & Outc & Outc & Outc & Applic & Outc & Outc & Applic & Outc & No & No \\
\hline $\mathrm{I} 05$ & More & More & More & More & All & All & All & All & All & All & All & One \\
\hline A03 & - & - & $\begin{array}{c}\text { Day or } \\
\text { Week }\end{array}$ & - & - & - & Day & Day & Day & - & - & - \\
\hline A04 & Unknown & Unknown & Known & Known & Partially & Partially & Partially & Partially & Known & Known & Partially & Known \\
\hline A05 & - & Int & Int & Int & Int & Int & Int & Int & Int & Int & Int & Ext \\
\hline $\mathrm{A} 06$ & - & - & $50-99$ & $0-19$ & $0-19$ & - & $20-49$ & $20-49$ & $20-49$ & $20-49$ & $0-19$ & $0-19$ \\
\hline N03 & - & - & Charged & Free & - & Free & Free & Free & Free & Free & Free & Free \\
\hline BPMM & $\mathrm{AOU}$ & ARY & SCC & $\mathrm{ABE}$ & MAU & ARM & BPM & BPM & O\&I & SKR & SPA & DET \\
\hline
\end{tabular}

\begin{tabular}{|c|c|c|c|c|c|c|c|c|c|}
\hline $\mathrm{I} 01$ & & & & $\overline{\text { Cluster A: }}$ & sic capabili & (continued & & & \\
\hline $\mathrm{I} 02$ & & & & & Both & & & & \\
\hline$\overline{\mathrm{I} 03}$ & Descr & & & & rescr & & & $\overline{\operatorname{Exp}}$ & prescr \\
\hline $\mathrm{I} 04$ & $\mathrm{SC}$ & & & & $\mathrm{SC}$ & & Collab & Generic & $\mathrm{SC}$ \\
\hline$\overline{\mathrm{A} 01}$ & Both & & & & & Both & Quali & Quali & Quali \\
\hline $\mathrm{A} 02$ & Subj & & & Subj & Both & Subj & Subj & Obj & Subj \\
\hline N01 & $\begin{array}{l}\text { Aware or } \\
\text { Bench }\end{array}$ & Awar & Bench & Aware & Aware & Aware & $\begin{array}{l}\text { Aware or } \\
\text { Bench }\end{array}$ & $\begin{array}{c}\text { Aware or } \\
\text { Bench or } \\
\text { Certif }\end{array}$ & Aware \\
\hline N02 & Outc & Applic & Outc & Applic & Applic & No & Outc & Applic & No \\
\hline $\mathrm{I} 05$ & More & All & All & More & More & More & More & More & More \\
\hline $\mathrm{A} 03$ & - & Day & - & - & - & Day & - & - & - \\
\hline A04 & Unknown & Known & Known & Partially & Known & Known & Partially & Known & Partially \\
\hline A05 & Int & Int & Int & Int & Int & Int & Int & Ext & Int \\
\hline A06 & $100-299$ & $0-19$ & $20-49$ & $0-19$ & $0-19$ & $20-49$ & $20-49$ & $50-99$ & $0-19$ \\
\hline N03 & - & Free & Free & - & - & Free & - & Charged & Free \\
\hline$\overline{\mathrm{BPMM}}$ & $\overline{I B M}$ & BIS & $\mathrm{MCC} 1$ & MAN & MCL & JER & SIM & ISO & $\overline{C G R}$ \\
\hline
\end{tabular}

For cluster B: basic capabilities + culture 


\begin{tabular}{|c|c|c|c|c|c|c|c|c|c|c|c|c|c|c|c|c|c|c|c|}
\hline$\overline{\mathrm{I} 01}$ & \multicolumn{19}{|c|}{ Cluster B: basic capabilities + culture } \\
\hline $\mathrm{I} 02$ & \multicolumn{10}{|c|}{ Continuous } & \multicolumn{9}{|c|}{ Staged } \\
\hline $\mathrm{I} 03$ & \multicolumn{4}{|c|}{ Descr } & \multicolumn{5}{|c|}{ Impl prescr } & Expl prescr & \multicolumn{3}{|c|}{ Descr } & \multicolumn{6}{|c|}{ Impl prescr } \\
\hline $\mathrm{I} 04$ & \multirow{2}{*}{\multicolumn{3}{|c|}{\begin{tabular}{c|c}
\multicolumn{2}{c}{ Generic } \\
Oyali
\end{tabular}}} & $\mathrm{SC}$ & \multirow{2}{*}{\multicolumn{4}{|c|}{$\begin{array}{l}\text { SC } \\
\text { Quali }\end{array}$}} & \multirow{2}{*}{$\begin{array}{c}\text { Collab } \\
\text { Ouali }\end{array}$} & Generic & \multirow{2}{*}{\multicolumn{2}{|c|}{\begin{tabular}{c|} 
Generic \\
Quali
\end{tabular}}} & \multirow{2}{*}{$\begin{array}{c}\text { SC } \\
\text { Both }\end{array}$} & \multicolumn{4}{|c|}{ Generic } & \multirow{2}{*}{$\begin{array}{r}\text { SC } \\
\text { Qual }\end{array}$} & \multirow{2}{*}{$\begin{array}{r}\text { Collab } \\
\text { Quali }\end{array}$} \\
\hline $\mathrm{A} 01$ & & & & Quali & & & & & & - & & & & $\mathrm{Qu}$ & & & 3oth & & \\
\hline $\mathrm{A} 02$ & Both & $\overline{B O}$ & & Both & \multicolumn{4}{|c|}{ Subj } & Subj & Subj & \multicolumn{2}{|c|}{ Both } & Subj & \multicolumn{2}{|c|}{ Subj } & \multicolumn{2}{|c|}{ Both } & $\mathrm{Si}$ & Subj \\
\hline N01 & Aware & \multicolumn{2}{|c|}{$\begin{array}{l}\text { Aware or } \\
\text { Bench }\end{array}$} & Aware & \multicolumn{2}{|c|}{ Aware } & Ber & & Aware & $\begin{array}{l}\text { Aware or } \\
\text { Bench }\end{array}$ & & are & $\begin{array}{l}\text { Aware or } \\
\text { Bench }\end{array}$ & $\begin{array}{r}\text { Awa } \\
\text { Ber }\end{array}$ & e or & & ware & $\mathrm{An}$ & Aware \\
\hline N02 & Applic & $\mathrm{App}$ & & Applic & Applic & Outc & $\mathrm{Ou}$ & & Applic & Outc & & $\mathrm{o}$ & Outc & Apr & & & pplic & $\mathrm{N}$ & Applic \\
\hline $\mathrm{I} 05$ & All & Mo & & More & More & More & $\overline{\mathrm{Mc}}$ & & One & All & & 11 & More & $\mathrm{A}$ & & & One & $\overline{\mathrm{M}}$ & One \\
\hline A03 & - & - & & Week & Day & - & - & & - & Day & $\begin{array}{l}\text { Da } \\
\text { We } \\
\text { Lo }\end{array}$ & $\begin{array}{l}\text { or } \\
\text { k or } \\
\text { ger }\end{array}$ & - & $\mathrm{D}$ & & & - & $\mathrm{D}$ & - \\
\hline$\overline{\mathrm{A} 04}$ & Unknown & Unkn & own & Partially & Partially & Known & $\overline{\mathrm{Kno}}$ & & Known & Unknown & Par & ially & Partially & $\overline{\mathrm{Kno}}$ & & & rtially & $\mathrm{Kn}$ & Known \\
\hline $\mathrm{A} 05$ & Int & In & & Int & Ext & Int & In & & Ext & Int & & t & Int & In & & & Int & & Int \\
\hline $\mathrm{A} 06$ & $20-49$ & - & & $100-299$ & $20-49$ & $20-49$ & $20-$ & & $20-49$ & $100-299$ & & 49 & - & $0-$ & & & $0-99$ & & $0-19$ \\
\hline $\mathrm{N} 03$ & Charged & Char & & Free & Free & Free & $\mathrm{Fr}$ & & Free & Charged & & $\overline{\mathrm{ee}}$ & - & $\mathrm{Fr}$ & & & arged & & - \\
\hline BPMM & CAM1 & ESI1 & & CAM3 & NET & MIC & MIC & & $\mathrm{RA}$ & DEL & HAR & & $\mathrm{CSC}$ & RUM & & SAP & & CHI & MAG \\
\hline $\mathrm{I} 01$ & & & & & & & ster B: basi & c capabilitie & ties + culture & (continued) & & & & & & & & & \\
\hline $\mathrm{I} 02$ & & & Stag & & & & & & & & Both & & & & & & & & \\
\hline I03 & & & $\overline{\text { Expl p1 }}$ & rescr & & & & & & Impl presc & & & & & & & Expl p & rescr & \\
\hline I04 & & & Colle & & & & & Generic & & & & & SC & & & & Coll & & \\
\hline A01 & & Qu & & & $\begin{array}{c}\text { Quanti or } \\
\text { Both }\end{array}$ & & & Quali & & & & & Quali & & $\mathrm{Bo}$ & & Bot & & \\
\hline $\mathrm{A} 02$ & Obj & $\mathrm{Su}$ & & Both & - & & & Subj & & & & & Subj & & $\mathrm{Bo}$ & & - & & \\
\hline N01 & Aware & $\mathrm{Aw}$ & & Aware & Aware & & Aw & & & Bench & & Aware & e or Bench & & $\begin{array}{r}\mathrm{Aw} \\
\text { or } \mathrm{B} \\
\end{array}$ & $\begin{array}{l}\text { are } \\
\text { nch }\end{array}$ & Awa & & \\
\hline N02 & Outc & $\mathrm{Ot}$ & tc & Outc & Outc & & Applic & & No & Applic & & & $\overline{\text { Outc }}$ & & $\mathrm{Ou}$ & & $\mathrm{Nc}$ & & \\
\hline I05 & One & One & More & One & One & & All & & All & All & & & More & & $\mathrm{Mc}$ & & $\mathrm{Mo}$ & & \\
\hline A03 & - & - & Day & - & - & & ek & Longer & Day & Week & & & - & & $\begin{array}{l}\text { Wee } \\
\text { Lon }\end{array}$ & & - & & \\
\hline $\mathrm{A} 04$ & Partially & Partially & Know & \begin{tabular}{l|l}
$\mathrm{vn}$ & Partially \\
\end{tabular} & Partially & Known & \begin{tabular}{|l|} 
Partially \\
\end{tabular} & Known & Known & Partially & & Known & & Partially & Kno & & Partic & & \\
\hline A05 & Int & Int & Ext & Int & Int & Int & Int & Int & Int & Int & & t & Ext & - & E. & & - & & \\
\hline A06 & - & - & $0-19$ & - & - & 0-19 & - & $0-19$ & $0-19$ & - & $\begin{array}{c}20- \\
49\end{array}$ & $50-99$ & $\begin{array}{l}20- \\
49\end{array}$ & 100-299 & 20 & & - & & \\
\hline $\mathrm{N} 03$ & - & - & Free & - & - & Charged & Charged & Charged & Free & Charged & - & Free & - & - & 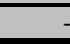 & & Fre & & \\
\hline$\overline{\text { BPMM }}$ & RAM & RAM & VIC & RAM & RAM & BPT & ROH & BPT & FIS & $\mathrm{ROH}$ & TOK & $\mathrm{MCC} 2$ & $\begin{array}{ll}\text { TOK } \\
\end{array}$ & PMG & $\mathrm{BOH}$ & & WOG & & \\
\hline
\end{tabular}

For cluster C: basic capabilities + culture + structure 


\begin{tabular}{|c|c|c|c|c|c|c|c|c|c|c|c|c|c|c|}
\hline$\overline{\mathrm{I} 01}$ & \multicolumn{14}{|c|}{ Cluster C: basic capabilities + culture + structure } \\
\hline $\mathrm{I} 02$ & \multicolumn{3}{|c|}{ Continuous } & \multicolumn{11}{|c|}{ Staged } \\
\hline $\mathrm{I} 03$ & \multicolumn{2}{|c|}{ Impl prescr } & Expl prescr & \multirow{2}{*}{\multicolumn{4}{|c|}{$\begin{array}{c}\text { Descr } \\
\text { Generic }\end{array}$}} & Impl prescr & \multicolumn{6}{|c|}{ Expl prescr } \\
\hline $\mathrm{I} 04$ & Generic & $\mathrm{SC}$ & Collab & & & & & Generic & \multirow{2}{*}{\multicolumn{3}{|c|}{ Quali }} & & & \\
\hline $\mathrm{A} 01$ & Quali & Quali & - & \multicolumn{4}{|c|}{ Quali } & Quali & & & & Quanti & \multicolumn{2}{|c|}{ Both } \\
\hline A02 & Subj & Subj & - & \multicolumn{4}{|c|}{ Subj } & Subj & $\begin{array}{l}\text { Obj or } \\
\text { Subj }\end{array}$ & \multicolumn{2}{|c|}{ Both } & - & \multicolumn{2}{|c|}{ Obj } \\
\hline N01 & Aware & Aware & Aware & \multicolumn{4}{|c|}{ Aware } & Aware & Aware & \multirow{2}{*}{\multicolumn{2}{|c|}{$\frac{\text { Aware }}{\text { No }}$}} & Aware & \multicolumn{2}{|c|}{ Aware } \\
\hline N02 & No & No & No & \multicolumn{3}{|c|}{ Applic } & No & Applic & No & & & No & Applic & No \\
\hline I05 & All & More & More & \multicolumn{3}{|c|}{ All } & All & $\begin{array}{l}\text { One or All } \\
\text { or Comb }\end{array}$ & One & One & All & One & More & One \\
\hline A03 & - & Day & - & \multicolumn{2}{|c|}{ Day } & $\begin{array}{l}\text { Week or } \\
\text { Longer }\end{array}$ & Day & - & - & - & - & - & $\begin{array}{l}\text { Week or } \\
\text { Longer }\end{array}$ & - \\
\hline$\overline{\mathrm{A} 04}$ & Known & Known & Partially & \multicolumn{2}{|c|}{$\begin{array}{l}\text { Partially } \\
\end{array}$} & $\begin{array}{l}\text { Partially } \\
\end{array}$ & $\begin{array}{l}\text { Partially } \\
\end{array}$ & Known & $\begin{array}{l}\text { Partially } \\
\end{array}$ & $\begin{array}{l}\text { Partially } \\
\end{array}$ & Unknown & $\begin{array}{l}\text { Partially } \\
\end{array}$ & Known & Partially \\
\hline $\mathrm{A} 05$ & - & Int & Int & \multicolumn{2}{|c|}{ Int } & Int & Int & Int & Int & Int & Int & Int & Ext & Int \\
\hline A06 & $0-19$ & $20-49$ & - & $20-49$ & $50-99$ & $50-99$ & $0-19$ & $100-299$ & - & - & - & - & $>=300$ & - \\
\hline $\mathrm{N} 03$ & Free & Free & - & Free & - & - & Free & Free & Free & Free & Charged & Free & Charged & Free \\
\hline BPMM & SCH1 & EKN & TAP & ORA & WIL & WIL & REM & HAM & $\overline{\text { GAR1 }}$ & GAR1 & CAM2 & $\overline{\text { GAR1 }}$ & OMG & GAR1 \\
\hline $\mathrm{I} 01$ & & & & luster C: bas & capabilities & culture + stru & re (continue & & & & & & & \\
\hline $\mathrm{I} 02$ & \multicolumn{4}{|c|}{ Staged } & \multicolumn{6}{|c|}{ Both } & & & & \\
\hline $\mathrm{I} 03$ & \multirow{2}{*}{\multicolumn{4}{|c|}{$\frac{\text { Expl prescr }}{\text { Generic }}$}} & \multicolumn{3}{|c|}{ Impl prescr } & \multicolumn{3}{|c|}{ Expl prescr } & & & & \\
\hline$\overline{\mathrm{I} 04}$ & & & & & & eric & $\mathrm{SC}$ & \multirow{2}{*}{\multicolumn{2}{|c|}{$\frac{\text { Generic }}{\text { Quali }}$}} & SC & & & & \\
\hline $\mathrm{A} 01$ & \multicolumn{4}{|c|}{ Both } & Quali & Both & Both & & & Quali & & & & \\
\hline $\mathrm{A} 02$ & Obj & & & Both & Both & Subj & Subj & Obj & Both & Subj & & & & \\
\hline N01 & $\begin{array}{l}\text { Bench or } \\
\text { Certif }\end{array}$ & & & Aware & $\begin{array}{l}\text { Aware or } \\
\text { Bench }\end{array}$ & $\begin{array}{c}\text { Aware or } \\
\text { Bench }\end{array}$ & $\begin{array}{l}\text { Aware or } \\
\text { Bench }\end{array}$ & $\begin{array}{c}\text { Aware or } \\
\text { Bench or } \\
\text { Certif }\end{array}$ & Aware & Aware & & & & \\
\hline N02 & Applic & & & No & Applic & Applic & Outc & Applic & Applic & No & & & & \\
\hline I05 & More & & & One & All & All & More & More & More & More & & & & \\
\hline A03 & $\begin{array}{l}\text { Week or } \\
\text { Longer }\end{array}$ & & & - & Longer & $\begin{array}{l}\text { Day or } \\
\text { Week }\end{array}$ & $\begin{array}{l}\text { Day or } \\
\text { Week }\end{array}$ & Longer & Longer & Day & & & & \\
\hline A04 & Known & Known & Partially & $\begin{array}{l}\text { Partially } \\
\end{array}$ & Unknown & Known & Known & Known & Known & Known & & & & \\
\hline A05 & Ext & Int & Int & Int & Int & Int & Ext & Int & Ext & Int & & & & \\
\hline A06 & $>=300$ & $100-299$ & - & - & $>=300$ & $0-19$ & 50-99 & $>=300$ & $100-299$ & $20-49$ & & & & \\
\hline $\mathrm{N} 03$ & Charged & Free & Free & Free & - & Charged & Free & Charged & Charged & Free & & & & \\
\hline BPMM & $\overline{\mathrm{OMG}}$ & HAR1 & GAR1 & GAR1 & ROS & IDS & CGF & SEI & FAA & LMI & & & & \\
\hline
\end{tabular}


Appendix E. Validation data

General information:

\begin{tabular}{|c|c|c|c|c|c|c|c|c|c|c|c|c|}
\hline General info & Perspective & \multicolumn{2}{|l|}{ Industry (NACE) } & $\begin{array}{l}\text { Org. size } \\
\text { (employees) }\end{array}$ & \multicolumn{2}{|c|}{$\begin{array}{l}\text { Annual turnover } \\
(\text { million } € \text { ) }\end{array}$} & \multicolumn{2}{|c|}{ Location } & Department & Position & $\begin{array}{l}\text { Org. } \quad \text { BPM } \\
\text { experience }\end{array}$ & $\begin{array}{l}\text { User BPM } \\
\text { experience }\end{array}$ \\
\hline Tester 1 & $\begin{array}{l}\text { Not yet } \\
\text { BPMM }\end{array}$ & \multicolumn{2}{|c|}{ Human health and social work } & 1-10 (micro) & \multicolumn{2}{|l|}{$<€ 2$ million } & \multicolumn{2}{|c|}{ Europe } & $\begin{array}{l}\text { Competence } \\
\text { centre }\end{array}$ & $\begin{array}{l}\begin{array}{l}\text { Lower } \\
\text { management }\end{array} \\
\end{array}$ & No & No notion \\
\hline Tester 2 & $\begin{array}{l}\text { Not yet } \\
\text { BPMM }\end{array}$ & \multicolumn{2}{|c|}{$\begin{array}{l}\text { Public administration, defence, compulsory } \\
\text { social security }\end{array}$} & $11-50$ (small) & \multicolumn{2}{|l|}{ No answer } & \multicolumn{2}{|c|}{ Europe } & Business & Operational & No & Theoretical \\
\hline Tester 3 & $\begin{array}{l}\text { Not yet } \\
\text { BPMM }\end{array}$ & \multicolumn{2}{|c|}{ Human health and social work } & $51-250$ (medium) & \multicolumn{2}{|l|}{ No answer } & \multicolumn{2}{|c|}{ Europe } & Business & $\begin{array}{l}\text { Lower } \\
\text { management }\end{array}$ & No & No notion \\
\hline Tester 4 & $\begin{array}{l}\text { Not yet } \\
\text { BPMM, but } \\
\text { quality MM }\end{array}$ & \multicolumn{2}{|c|}{$\begin{array}{l}\text { Public administration, defence, compulsory } \\
\text { social security }\end{array}$} & $1,001-5,000$ (large) & \multicolumn{2}{|l|}{$€ 10-50$ million } & \multicolumn{2}{|c|}{ Europe } & Business & $\begin{array}{l}\text { Middle } \\
\text { management }\end{array}$ & $\begin{array}{l}\text { Yes, in some } \\
\text { parts of the } \\
\text { organisations }\end{array}$ & Theoretical \\
\hline User 1 & $\begin{array}{l}\text { Not yet } \\
\text { BPMM }\end{array}$ & \multicolumn{2}{|c|}{$\begin{array}{l}\text { Manufacturing of products, e.g. equipment, } \\
\text { chemicals, pharmaceuticals, installations }\end{array}$} & $501-1,000$ (large) & \multicolumn{2}{|c|}{$>€ 50,000$ million } & $\begin{array}{l}\text { Europ } \\
\text { North } \\
\text { Ameri }\end{array}$ & $\begin{array}{l}\text { \& South } \\
\text { a, Asia }\end{array}$ & Business & $\begin{array}{l}\text { Middle } \\
\text { management }\end{array}$ & $\begin{array}{l}\text { Yes, in some } \\
\text { parts of the } \\
\text { organisation }\end{array}$ & No notion \\
\hline User 2 & $\begin{array}{l}\text { Already } \\
\text { BPMM }\end{array}$ & $\begin{array}{l}\text { Public administrati } \\
\text { social security }\end{array}$ & n, defence, compulsory & $5,001-10,000$ (large) & $€ 50-500$ million & & Europ & & $\begin{array}{l}\text { Competence } \\
\text { centre }\end{array}$ & $\begin{array}{l}\text { Lower } \\
\text { management }\end{array}$ & $\begin{array}{l}\text { Yes, in some } \\
\text { parts of the } \\
\text { organisation }\end{array}$ & $\begin{array}{l}\text { Both, } \\
\text { theoretical } \\
\text { and empirical }\end{array}$ \\
\hline User 3 & Researcher & $\begin{array}{l}\text { Professional, sci } \\
\text { activities }\end{array}$ & ntific and technical & $5,001-10,000$ (large) & $€ 500-1,000$ mill & & Europ & & Business & Operational & No & Theoretical \\
\hline forma & garding & e requireme & & & & & & & & & & \\
\hline Requirement & & & & Design product & & & & & & & Design process & \\
\hline & Effectiveness & & Utility & & & & Effic & iency & Quality & & & \\
\hline & BPMM after & BPMM before & Use of BPMM after & \begin{tabular}{l}
\multicolumn{2}{l}{ Satisfaction } \\
with BPMM \\
after
\end{tabular} & $\begin{array}{l}\text { Recommend } \\
\text { to others? }\end{array}$ & $\begin{array}{l}\text { Time } \\
(\min \end{array}$ & $\begin{array}{l}\text { spent } \\
\text { utes) }\end{array}$ & $\begin{array}{l}\text { Satisfactic } \\
\text { with tin } \\
\text { spent } \\
\end{array}$ & \begin{tabular}{l|l} 
on & $\begin{array}{l}\text { Satisfaction } \\
\text { ne } \\
\text { with out } \\
\text { presentatio }\end{array}$ \\
\end{tabular} & 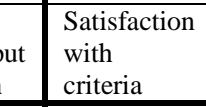 & $\begin{array}{l}\text { Satisfaction } \\
\text { with question } \\
\text { formulations }\end{array}$ & $\begin{array}{l}\text { Satisfaction } \\
\text { with } \\
\text { sequence } \\
\end{array}$ \\
\hline Tester 1 & BIS & None & Maybe, no decision power & 4 & Yes & 30 & & 6 & 7 & 4 & 4 & 4 \\
\hline Tester 2 & ISO & None & No, no decision power & 6 & Yes & 23 & & 6 & 4 & 6 & 6 & 6 \\
\hline Tester 3 & ISO & None & No, no decision power & 6 & Yes & 25 & & 6 & 6 & 6 & 7 & 6 \\
\hline Tester 4 & $\mathrm{ROH}$ & EFQM (quality) & Yes & 4 & $\begin{array}{l}\text { No, first tool } \\
\text { improvements }\end{array}$ & 30 & & 6 & 4 & 4 & 4 & 4 \\
\hline User 1 & CGF & None & Yes & $\begin{array}{l}\text { 4, first using } \\
\text { the BPMM }\end{array}$ & $\begin{array}{l}\text { No answer, } \\
\text { too early }\end{array}$ & 15 & & 6 & 6 & 5 & 5 & 6 \\
\hline User 2 & HAM & WIL & Yes & 6 & Yes & 15 & & 6 & 6 & 6 & 6 & 6 \\
\hline User 3 & HAM & MCC1 & Maybe & 6 & Yes & 21 & & 7 & 7 & 6 & 6 & 6 \\
\hline
\end{tabular}

